\title{
Automated Assessment of Blood Flow in the Cardiovascular System Using 4D Flow MRI
}

Mariana Bustamante

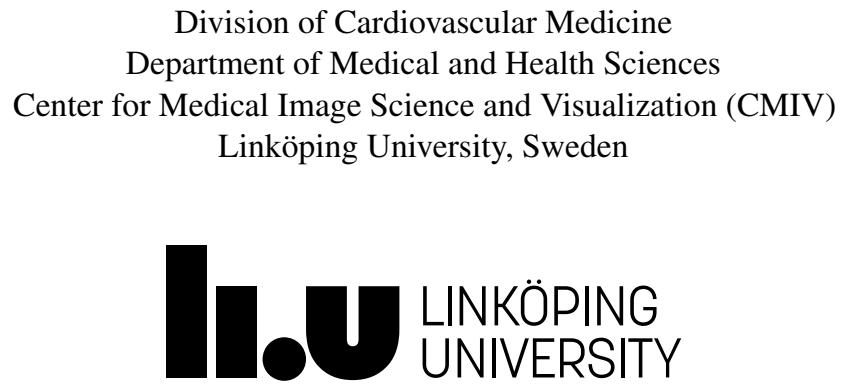




\section{Automated Assessment of Blood Flow in the Cardiovascular System Using 4D Flow MRI}

Linköping University Medical Dissertations

Dissertation No. 1613

Department of Medical and Health Sciences

Linköping University

SE-581 83, Linköping, Sweden

http://www.liu.se/cmr

Printed by:

LiU-Tryck, Linköping, Sweden

ISBN 978-91-7685-346-7

ISSN 0345-0082

Copyright ( 2018 Mariana Bustamante, unless otherwise noted

No part of this publication may be reproduced, stored in a retrieval system, or be transmitted, in any form or by any means, electronic, mechanic, photocopying, recording, or otherwise, without prior permission of the author.

Cover: Instantaneous streamline visualization of the thoracic and cardiac blood flows at different phases of the cardiac cycle for five datasets.

Flip book: Segmentation of the cardiac chambers and great thoracic vessels over the cardiac cycle. 




\section{Abstract}

Medical image analysis focuses on the extraction of meaningful information from medical images in order to facilitate clinical assessment, diagnostics and treatment. Image processing techniques have gradually become an essential part of the modern health care system, a consequence of the continuous technological improvements and the availability of a variety of medical imaging techniques.

Magnetic Resonance Imaging (MRI) is an imaging technique that stands out as non-invasive, highly versatile, and capable of generating high quality images without the use of ionizing radiation. MRI is frequently performed in the clinical setting to assess the morphology and function of the heart and vessels. When focusing on the cardiovascular system, blood flow visualization and quantification is essential in order to fully understand and identify related pathologies. Among the variety of MR techniques available for cardiac imaging, 4D Flow MRI allows for full threedimensional spatial coverage over time, also including three-directional velocity information. It is a very powerful technique that can be used for retrospective analysis of blood flow dynamics at any location in the acquired volume.

In the clinical routine, however, flow analysis is typically done using twodimensional imaging methods. This can be explained by their shorter acquisition times, higher in-plane spatial resolution and signal-to-noise ratio, and their relatively simpler post-processing requirements when compared to 4D Flow MRI. The extraction of useful knowledge from 4D Flow MR data is especially challenging due to the large amount of information included in these images, and typically requires substantial user interaction.

This thesis aims to develop and evaluate techniques that facilitate the postprocessing of thoracic 4D Flow MRI by automating the steps necessary to obtain hemodynamic parameters of interest from the data. The proposed methods require little to no user interaction, are fairly quick, make effective use of the information available in the four-dimensional images, and can easily be applied to sizable groups of data. The addition of the proposed techniques to the current pipeline of 4D Flow MRI analysis simplifies and expedites the assessment of these images, thus bringing them closer to the clinical routine. 



\section{Populärvetenskaplig Beskrivning}

Medicinsk bildanalys fokuserar på extrahering av meningsfull information från medicinska bilder för att underlätta klinisk bedömning, diagnostik, och behandling. Bildbehandlingsteknik har gradvis blivit en viktig del av det moderna sjukvårdsystemet, en följd av de kontinuerliga tekniska förbättringarna och tillgången till en mängd olika medicinska bildtekniker.

Magnetic resonanstomografi (MRT) är en bildteknik som är ickeinvasiv, flexibel och kan generera bilder av hög kvalitet utan joniserande strålning. MRT utförs ofta i klinisk miljö för att bedöma anatomi och funktion av hjärtat och blodkärlen. När man fokuserar på hjärt-kärlsystemet är bedömning av blodflödet viktigt för att kunna förstå och identifiera sjukdomar fullt ut. Bland de olika MRT-teknikerna som är tillgängliga för avbildning av hjärtat möjliggör 4D flödes-MRT komplett täckning av hjärtat i tre dimensioner över tid, och med hastighetsinformation i tre riktningar. 4D flödes-MRT är en mycket effektiv metod som kan användas för retrospektiv analys av blodflödesdynamik på vilken position som helst i den avbildade volymen.

Till vardags görs dock blodflödesanalysen vanligtvis på bilder tagna med tvådimensionella avbildningsmetoder. Detta kan förklaras av deras kortare insamlingstider, högre spatiella upplösning, bättre signal-brusförhållandet, och att de är relativt enklare att efterbehandla jämfört med 4D flödes-MRT. Utvinningen av användbar information från 4D flödes-MRT-data är väldigt utmanande på grund av den stora mängden information som dessa bilder innehåller och kräver vanligtvis väsentlig användarinteraktion.

Denna avhandling syftar till att utveckla och utvärdera metoder som underlättar efterbehandlingen av 4D flödes-MRT genom att automatisera de steg som är nödvändiga för att härleda hemodynamiska parametrarna av intresse från dessa data. De föreslagna metoderna kräver liten eller ingen användarinteraktion, är relativt snabba, använder all information som finns i de fyrdimensionella bilderna, och kan enkelt appliceras på stora datamängder. Tillägget av de i avhandlingen beskrivna metoderna till den nuvarande analysen av 4D flödes-MRT medger en avsevärd förenkling och uppsnabbad utvärdering, vilket gör att den avancerade 4D flödes MRT-tekniken kommer närmare att kunna användas i kliniskt rutinarbete. 



\section{Acknowledgements}

The time that I've spent in Linköping working on the contents of this dissertation have been incredibly fun, and I consider myself lucky to have had the opportunity to do this project. During the past five years I've been surrounded by a talented group of people that have kindly guided and assisted me in completing this thesis, and to whom I owe a lot of gratitude.

First of all I would like to acknowledge my supervisor Tino Ebbers for the great ideas, the trust, advice, and for keeping me focused on the long term goals. To my co-supervisors Carl-Johan Carlhäll and Petter Dyverfeldt for their guidance and patience in answering my many questions.

I am also very grateful to my coworkers at the Cardiovascular Magnetic Resonance group and the Center for Medical Image Science and Visualization. Thanks to Belén Casas, who has been working side by side with me since the beginning of our experience in the academia, for the talks and the company. To Vikas Gupta and Merih Cibis, for sharing their knowledge and experience with me, and being always open to discuss new ideas. To Jonatan Ericsson, Sven Petersson, Jonas Lantz, Alexandru Fredriksson, Jakub Zajak, Sofia Kvernby, Magnus Ziegler, Federica Viola, and Hojin Ha, for the many fikas, fruitful discussions, and lunch adventures.

To my small but strong group of venezuelan friends in Sweden: Harold, Elio, Verónica, María Andreína, and Oscar, for the hallacas, the empanadas, and the good times.

Thank you Óli, for moving to a new city with me without a second thought when I got this opportunity, and for supporting me every day. Thank you also to Unnur, Sveinn, Ásta, Ylfa, and the rest of my Icelandic family, for being so welcoming and kind to me.

Para Dorina, Wilmer, Ramona, y mi familia en Venezuela.

Mariana

Linköping, March 2018 



\section{Funding}

This work has been conducted in collaboration with the Center for Medical Image Science and Visualization (CMIV) at Linköping University, Sweden. CMIV is acknowledged for provision of financial support and access to leading edge research infrastructure. The author also acknowledges the financial support provided by:

- The European Union's Seventh Framework Programme (FP7/2007-2013) under grants:

(a) 310612, project HEART4FLOW.

(b) 223615, project DOPPLER-CIP.

- The Swedish Research Council, under grant number 621-2014-6191.

- The Swedish Heart and Lung Foundation, under grant number 20140398.

- The Knut and Alice Wallenberg foundation, under grant number KAW 2013.0076. 



\section{List of Papers}

This thesis is based on the following papers, which will be referred to by their Roman numerals:

I. Improving Left Ventricular Segmentation in Four-dimensional Flow MRI using Intramodality Image Registration for Cardiac Blood Flow Analysis.

Vikas Gupta $^{\dagger}$, Mariana Bustamante ${ }^{\dagger}$, Alexandru Fredriksson, Carl-Johan Carlhäll and Tino Ebbers.

Magnetic Resonance in Medicine, 2018, 79:554.

II. Atlas-based Analysis of 4D Flow CMR: Automated Vessel Segmentation and Flow Quantification.

Mariana Bustamante, Sven Petersson, Jonatan Eriksson, Urban Alehagen, Petter Dyverfeldt, Carl-Johan Carlhäll and Tino Ebbers.

Journal of Cardiovascular Magnetic Resonance, 2015, 17:87.

III. Improving Visualization of 4D Flow CMR with Four-dimensional Angiographic Data: Generation of a 4D Phase-Contrast Magnetic Resonance CardioAngiography (4D PC-MRCA).

Mariana Bustamante, Vikas Gupta, Carl-Johan Carlhäll and Tino Ebbers. Journal of Cardiovascular Magnetic Resonance, 2017, 19:47.

IV. Creating Hemodynamic Atlases of Cardiac 4D Flow MRI.

Merih Cibis, Mariana Bustamante, Jonatan Eriksson, Carl-Johan Carlhäll and Tino Ebbers.

Journal of Magnetic Resonance Imaging, 2017, 46:5.

V. Automated Multi-atlas Segmentation of Cardiac 4D Flow MRI.

Mariana Bustamante, Vikas Gupta, Daniel Forsberg, Carl-Johan Carlhäll, Jan Engvall and Tino Ebbers.

Submitted for journal publication, 2018.

$\dagger$ These authors contributed equally to this work. 



\section{Abbreviations}

2D two-dimensional

3D three-dimensional

4D four-dimensional

b-SSFP balanced Steady-State Free Precession

CE-MRA Contrast Enhanced Magnetic Resonance Angiography

DSC Dice Similarity Coefficient

ED End-diastole

EDV End-diastolic volume

EF Ejection Fraction

ES End-systole

ESV End-systolic volume

H Helicity

HD Hausdorff Distance

KE Kinetic Energy

LA Left atrium

LV Left ventricle

MIP Maximum Intensity Projection

MRA Magnetic Resonance Angiography

MRI Magnetic Resonance Imaging

NCC Normalized Cross Correlation

NMI Normalized Mutual Information 
PC-MRA Phase Contrast MRA

PC MRCA Phase Contrast Magnetic Resonance CardioAngiography

PC-MRI Phase Contrast Magnetic Resonance Imaging

RA Right atrium

RF Radio frequency

ROI Region of Interest

RV Right ventricle

SSD Sum of Squared Differences

SV Stroke Volume

TKE Turbulent Kinetic Energy

VENC Velocity encoding range 


\section{Contents}

Abstract

Populärvetenskaplig Beskrivning vii

$\begin{array}{ll}\text { Acknowledgements } & \text { ix }\end{array}$

Funding

List of Papers $\quad$ xiii

Abbreviations $\quad$ Xv

1 Introduction 1

2 Aims 3

3 Physiological Background 5

3.1 The Cardiovascular System . . . . . . . . . . . . . . . 5

3.2 Cardiovascular Blood Flow . . . . . . . . . . . . . 5

4 Cardiovascular Magnetic Resonance Imaging 9

4.1 Magnetic Resonance Imaging Basics . . . . . . . . . . . . . . . . . . . . . . . 9

4.2 MRI of the Cardiovascular System . . . . . . . . . . . . . . . 9

4.3 Balanced Steady-State Free Precession . . . . . . . . . . . . . . 10

4.4 4D Flow MRI . . . . . . . . . . . . . . . . . . . . . . . . . . . . . . . . . 12

4.5 MR Angiography . . . . . . . . . . . . . . . . . . 13

4.5.1 Contrast-enhanced MRA (CE-MRA) . . . . . . . . . 13

4.5.2 Phase-contrast MRA (PC-MRA) . . . . . . . . . . . . 14

4.5.3 4D Flow MRI generated angiography . . . . . . . . . . 14

5 Medical Image Analysis 17

5.1 Image Registration . . . . . . . . . . . . . . . . . . 17

5.1.1 Registration Considerations for this Thesis . . . . . . . . 19

5.2 Image Segmentation . . . . . . . . . . . . . . . . 23

5.2.1 Atlas and Multi-atlas Segmentation . . . . . . . . . . 23

5.2.2 Evaluating Segmentation . . . . . . . . . . . 25

6 4D Flow MRI Assessment 29

6.1 Vessel Segmentation . . . . . . . . . . . . . . . . . . . . . . . . . 29

6.2 Cardiac Segmentation . . . . . . . . . . . . . . . . . . . 29

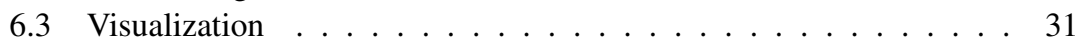

6.4 Hemodynamic Analysis . . . . . . . . . . . . . . . . 31 
7 Proposed Techniques 33

7.1 Automatic Segmentation Correction . . . . . . . . . . . . . . 33

7.2 Vessel Segmentation . . . . . . . . . . . . . . . . 37

7.3 Angiographic Visualization . . . . . . . . . . . . . . . . 42

7.4 Assessment of Hemodynamic Parameters . . . . . . . . . . . . . . 45

7.5 Cardiac Segmentation . . . . . . . . . . . . . . 48

8 Summary and Future Outlook 5

8.1 Contributions of this thesis . . . . . . . . . . . . . 57

8.2 Comments on the Current Implementation . . . . . . . . . . . . 57

8.3 Evaluation of Results . . . . . . . . . . . . . . . . . . 58

8.4 Use of Contrast Agents . . . . . . . . . . . . . . . . . . . . 59

8.5 Future Outlook .................... 60 


\section{Chapter 1}

\section{Introduction}

Changes in intracardiac blood flow patterns appear to be early markers of cardiac disease. Altered blood flow patterns have been observed in many cardiac diseases, often as a result of early remodeling of the cardiac chambers. These alterations have turned out to be extremely difficult to predict based on anatomy [1].

Magnetic Resonance Imaging (MRI) is a very versatile technique, allowing for velocity imaging at any location and in any direction without harmful radiation. For non-invasive flow measurements, MRI is often seen as the gold standard, particularly since it can be used to generate flow measurements with good spatial resolution in two or three dimensions [2].

Assessment and quantification of blood flow velocities in the entire cardiovascular system has been achieved successfully using 4D Flow MRI [3]. This technique enables the calculation of hemodynamic markers such as flow volumes, flow eccentricity, pulse wave velocity, pressure, turbulence, wall shear stress, among others, which can be used to provide a greater understanding of abnormal flows in the cardiovascular system. Visualization of these markers, typically through volume renderings or isosurfaces, and of the blood flow data using vector plots or particle traces, can also be of relevance during cardiac function assessment.

Manual analysis of 4D Flow MR data has been shown to be extremely difficult and time-consuming. Therefore, current methods for 4D Flow MRI analysis have included some degree of automation $[4,5]$. The bulk of the studies, however, continue to rely on manual methods, especially when it comes to segmentation of the heart's chambers, necessary for the calculation of several important hemodynamic markers.

Due to the large amount of information included in a 4D Flow MRI acquisition, manual methods applied on them are tedious and usually very time consuming. The aim of this project is to develop and evaluate tools for the assessment of 4D Flow MRI data that can be used in large groups of data, are mostly automatic, and consequently easy to use. Moreover, the formulated tools should be able to take advantage of all the information available in the 4D Flow MRI dataset.

Image analysis offers an extensive array of techniques with the potential to automate many of the steps required in order to extract useful information from medical images. Appropriate application of these techniques whenever possible is increasingly necessary in the modern health care system due to the continued growth 
of the amount of images acquired daily for medical purposes. A trend that will most likely continue with the ongoing advances in image generating techniques, together with the increase in computing power available for image processing. With this in mind, the focus of this work lies in the application and evaluation of current image processing techniques to improve 4D Flow MR-based research.

This thesis is outlined as follows: The main aims of the project are summarized in Chapter 2. A physiological background of the cardiovascular system and its blood flow is included in Chapter 3. Chapter 4 contains descriptions of the most important MRI techniques used during throughout this project, while Chapter 5 provides an introduction to the image analysis techniques applied in this work. Chapter 6 describes the current state of the art for 4D Flow MRI assessment, focusing on each of the steps required in order to obtain useful information from this type of MR acquisition. Subsequently, Chapter 7 presents a series of proposed improvements to this standard developed and evaluated during the course of this project. Chapter 8 concludes with a summary of the goals achieved and a future outlook for this field of research. 


\section{Chapter 2}

\section{Aims}

The main objective of this work was to develop tools to improve the clinical utility of the 4D Flow MRI acquisition technique. More specifically, we focused on the following aims:

- Improving the analysis process of 4D Flow MR images in order to facilitate the assessment of blood flow in the cardiovascular system.

- Decreasing the time necessary to analyze 4D Flow MR images by automating steps that typically require arduous manual supervision.

- Improving visualization of vessels and blood pool areas when using 4D Flow MRI data, with special focus on including all the information present in the four-dimensional images.

- Increasing the feasibility of 4D Flow MRI data analysis in sizable cohorts, for both individual and group-wise assessment of the images. 



\section{Chapter 3}

\section{Physiological Background}

\subsection{The Cardiovascular System}

The main purpose of the cardiovascular system is to drive and maintain the circulation of blood throughout the body. It serves to provide the cells with oxygen and nutrients, while also facilitating the management of waste products in the opposite direction.

The cardiovascular system's main component is the heart, which functions as a pump that drives the circulation of blood through the body by way of the vascular system. The heart itself is composed of two pumps with equalized outputs; the left heart which pumps oxygenated blood, and the right heart which handles deoxygenated blood.

Anatomically, the vascular system can also be classified into two circuits comprising the systemic and the pulmonary circulation. The systemic circulation carries oxygenated blood from the cardiac left ventricle through the arteries to all the body tissues, and returns deoxygenated blood through the veins back to the right atrium. The pulmonary circulation handles the delivery of blood to and from the lungs by transporting deoxygenated blood from the right ventricle to the lungs, and from there, newly oxygenated blood is returned to the left atrium via the pulmonary veins [6]. The cardiovascular system is illustrated in Figure 1. A detailed image of the heart can be seen in Figure 2.

\subsection{Cardiovascular Blood Flow}

Each chamber of the heart has a specific size and shape that influences the way in which the blood moves through it with each heart beat. Previous studies have proposed that the specific distribution of each of the cardiac chambers with respect to each other, together with the way that each of them contracts during the cardiac cycle, contribute to the conservation of energy in the flowing blood, therefore improving the heart's efficiency [7, 8].

There has been significant research establishing the fact that different pathologies cause alterations in the normal cardiac flow patterns [9-11]. Consequently, visualization and quantification of altered flows could also be used as an additional 


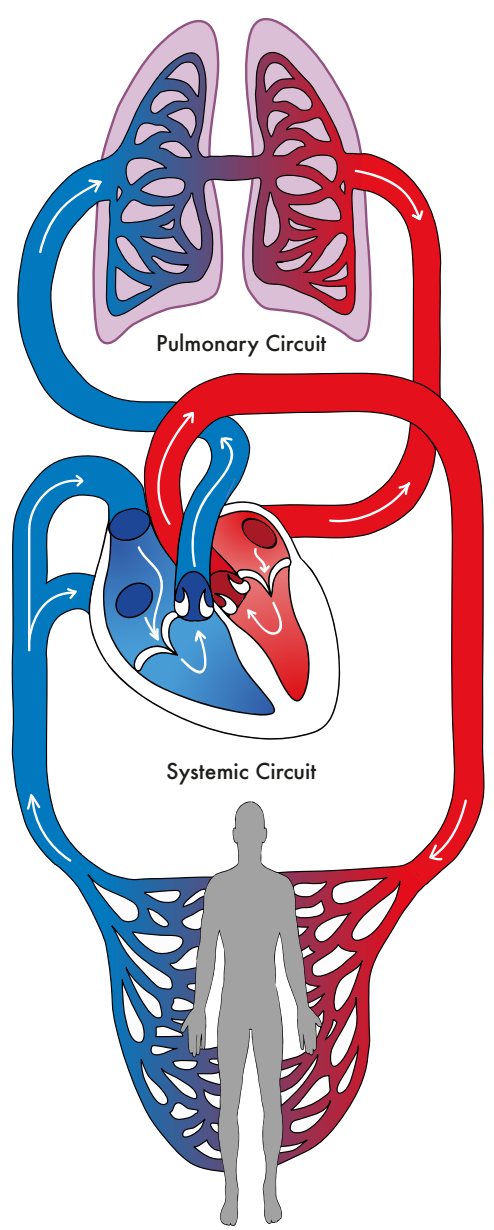

Figure 1: Schema of the cardiovascular system depicting the systemic and pulmonary circuits, the white arrows indicate the direction of blood flow. Red: Oxygen rich blood, blue: Carbon dioxide rich blood.

diagnostic tool in cardiovascular research. However, flow pattern analysis relies strongly on visualization; therefore, most of the techniques require a subjective analysis of the flow information, which in many cases is not quantitative. Clinically useful, concise information can be difficult to generate.

In practice, providing a per-case analysis of flow information has been of value in specific cases, particularly when the morphology observed is greatly removed from the norm, or when the flow patterns have been significantly altered by the underlying pathology. Recently, however, studies have applied MRI techniques to generate information that represents a group of subjects in a single dataset $[12,13]$, which could help in identifying the characteristic flow patterns of particular diseases. 


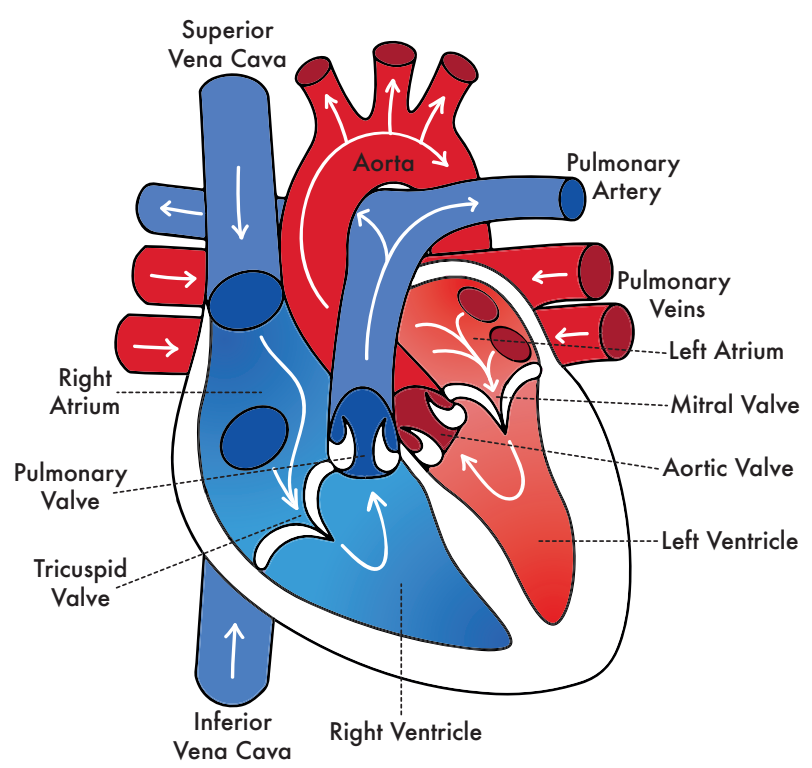

Figure 2: Anatomy of the human heart. Red: Left side of the heart containing oxygen rich blood, blue: Right side of the heart with deoxygenated blood. The white arrows indicate the direction of blood flow.

There has also been some discussion about the idea of using flow analysis to help in the generation of treatments for diseases that affect the normal flow patterns in the heart and vessels [1]. For example, valve replacements and stents that focus on the preservation of the original flow patterns of the affected region might produce better results than valves that simply focus on restoring flow to the ventricle or vessel $[14,15]$. Another way of utilizing blood flow pattern information for disease diagnostics has been proposed by Eriksson et al. [16]. This semi-automatic method can be used to analyze and quantify the different components of the cardiac flow, and has been evaluated for the left and right ventricles [17-19], and has also been modified to adapt to the left atrial flow [20].

In order to improve diagnosis, treatment, and follow up of cardiac diseases, we need to be able to quantify the severity of cardiac dysfunction, and to understand the stages and processes that can provoke heart failure. The incorporation of flow-based assessments into the diagnostic and treatment strategies for cardiovascular diseases would positively influence the ability of the physicians to understand the underlying physiological alterations caused by these disorders. It is our intent to eventually reach a point where the assessment of flow patterns will become just one of the available visualizations rendered directly after a medical examination. With the steady improvement in medical imaging techniques, and the increasing computing power available to technicians and researchers this idea might soon become a reality. 



\section{Chapter 4}

\section{Cardiovascular Magnetic Resonance Imaging}

\subsection{Magnetic Resonance Imaging Basics}

This section aims to explain the basic concepts behind Magnetic Resonance Image, for a more detailed perspective, please refer to [21, 22].

Magnetic Resonance Imaging is a medical imaging technique based on the fact that the angular momentum, or spin, of hydrogen protons contained in biological tissues, can interact with an external magnetic field. When placed in a strong magnetic field, $\overrightarrow{B_{0}}$, the proton's spin will precess about the field's direction. Using a radio frequency (RF) pulse, an MRI scanner is able to influence the magnetization alignment of the hydrogen spins relative to $\overrightarrow{B_{0}}$. Subsequently, the protons return to equilibrium towards the direction of $\overrightarrow{B_{0}}$ at different speeds depending on the proton density of the tissue to which they belong.

The time that it takes for the magnetization to return to equilibrium is called relaxation time, two types of which are of interest in MR imaging: The longitudinal relaxation time, typically represented by a time constant $T_{1}$, follows the recovery of the magnetization vector component in the direction of $\overrightarrow{B_{0}}$; and the transversal relaxation time, represented by $T_{2}$, follows the relaxation of the transverse component of the magnetization vector, related to the loss of phase coherence of the hydrogen spins.

These changes in magnetization are exploited by a combination of RF pulses and gradient fields resulting in a signal that can be detected by a receiver coil in the MR camera. Using these tools, the MR scanner is also able to locate each received signal to a specific position in the resulting raw data. Finally, a Fourier transform is applied to the data, generating an image in which the intensity value of each element is related to the relaxation times of the different tissues included in the picture.

\subsection{MRI of the Cardiovascular System}

Magnetic Resonance Imaging of the heart and vessels is commonly used in the clinic with a variety of aims, such as assessment of cardiac anatomy and structure, 
measurement of myocardial function, tissue characterization, visualization and quantification of myocardial perfusion, and visualization and quantification of vascular and intracardiac blood flow. With these goals in mind, several MR acquisition techniques have been specifically developed and extended for use in the cardiovascular system.

A major challenge when imaging the heart and thoracic vessels using MR is the likelihood of motion artifacts in the resulting images. Therefore, successful cardiac imaging requires handling two types of motion: the motion of the heart as it pumps, and the motion of the thorax caused by respiration. Cardiac motion compensation is achieved by synchronizing the image acquisition to the signal of an electrocardiogram or photoplethysmograph placed on the subject. Using this technique, the acquisition sequence is able to identify the phase of the cardiac cycle to which each acquired segment belongs to. Respiratory motion can be managed with the use of breath-held or respiratory-triggered acquisitions. In cardiac imaging, it is common to acquire one section (typically a slice) of the image per breathhold. Other acquisition methods handle respiratory motion by locating a respiratory tracking device on the subject's abdomen, or by using a pencil beam, usually called respiratory navigator, to acquire a column of pixels at the interface between the lung and the liver [23].

The following sections describe the MR acquisition methods most significant for this work.

\subsection{Balanced Steady-State Free Precession}

Balanced steady-state free precession (b-SSFP) is an MRI acquisition technique typically chosen for cardiac anatomy visualization and cardiac function assessment due to its relatively short acquisition times, very high signal-to-noise ratio, and its ability to result in images with high contrast between blood and myocardium. One of the most important characteristics of b-SSFP MR images is that their contrast is given by a composition of $T_{1}$ and $T_{2}$ contributions; this is advantageous when imaging tissues with different ratios of $T_{1}$ and $T_{2}$, such as blood and muscle, fat and muscle, or vessels and surrounding tissue. This feature makes b-SSFP MR a very good choice for assessing myocardial motion and performing angiographic imaging [24].

b-SSFP MR allows for the acquisition of 2D and 3D cine (time-resolved) images that include the whole cardiac cycle. A typical b-SSFP acquisition for cardiac assessment is composed of 2D long-axis cine images containing two-, three-, and four-chamber views of the heart, and a stack of 2D short-axis cine images to cover the cardiac ventricles, with spatial resolution of about $1 \mathrm{~mm}^{2}$, and slice thickness of $7-10 \mathrm{~mm}$. Figure 3 shows the location of the standard cardiac imaging views relative to the cardiac anatomy. Figure 4 contains an example of a b-SSFP cardiac MRI.

Each image, or subset of images is, when possible, acquired during a breath-hold. Performing the image acquisition at the end of exhalation during a breath-hold has been determined to be more consistent, however, misalignments between the slices 
of the short-axis stack caused by slightly different positions during breath-holding are a common issue in cardiac MR imaging [25].

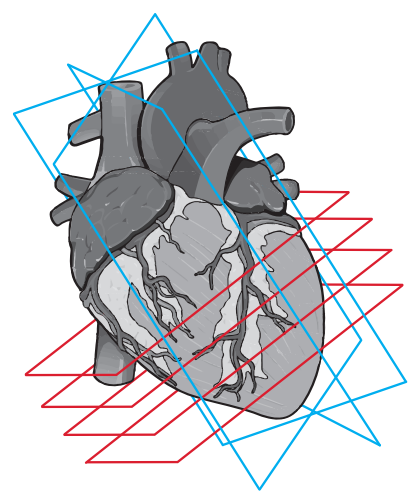

Figure 3: Location of the cardiac imaging planes relative to the anatomy of the heart. Red: Short-axis views, blue: Long-axis views.
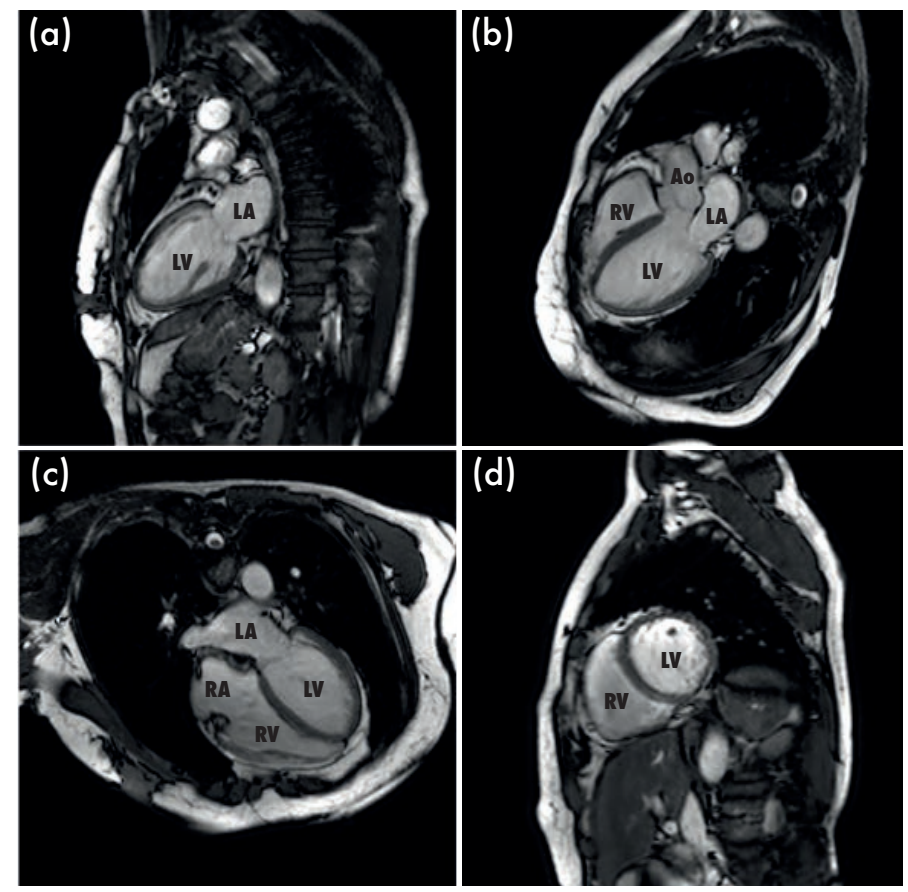

Figure 4: Cardiovascular MRI using balanced steady-state free precession. (a) Long-axis two-chamber view, (b) long-axis three-chamber view, (c) long-axis four-chamber view, and (d) short-axis view typically composed of a stack of images. 


\subsection{D Flow MRI}

Blood flow patterns in the heart have been studied using a variety of medical imaging techniques such as echocardiography, particle image velocimetry, and Magnetic Resonance Imaging [26]. These imaging techniques are able to provide in-vivo visualizations of the cardiac blood flow patterns, in addition to quantifiable flow velocity information. Among them, MRI permits two- and three-dimensional visualization of blood flow dynamics, and is typically considered the clinical gold standard [2].

In the clinical routine, the most common flow imaging technique based on magnetic resonance is Phase Contrast MRI (PC-MRI). This acquisition makes use of an initial gradient field followed by an opposing field, usually called bipolar gradients, which cause phase shifts in the flowing spins while the static tissue's phase remains unaffected. Furthermore, the phase shift accumulated by the moving tissues will be proportional to the velocity in the gradients' direction. Consequently, the blood flow velocity can be visualized and quantified in the resulting images [27-29].

The relationship between blood velocity and the phase shift is calibrated by a parameter called velocity encoding range or VENC. The VENC value indicates the velocity that corresponds to a phase shift of $\pi$ radians. VENC is the maximum velocity (positive or negative) that can be properly encoded by the sequence, and velocities higher than $\pm \mathrm{VENC}$ will result in aliasing on the acquired image. The chosen VENC must be able to encode the highest expected velocities, but also selecting a too high VENC will result in a large dynamic range where lower velocities will be more difficult to observe. Consequently, choosing a proper VENC value to encode the required velocities in the resulting image is of great importance, and it is typically done based on the visualization requirements of the specific case.

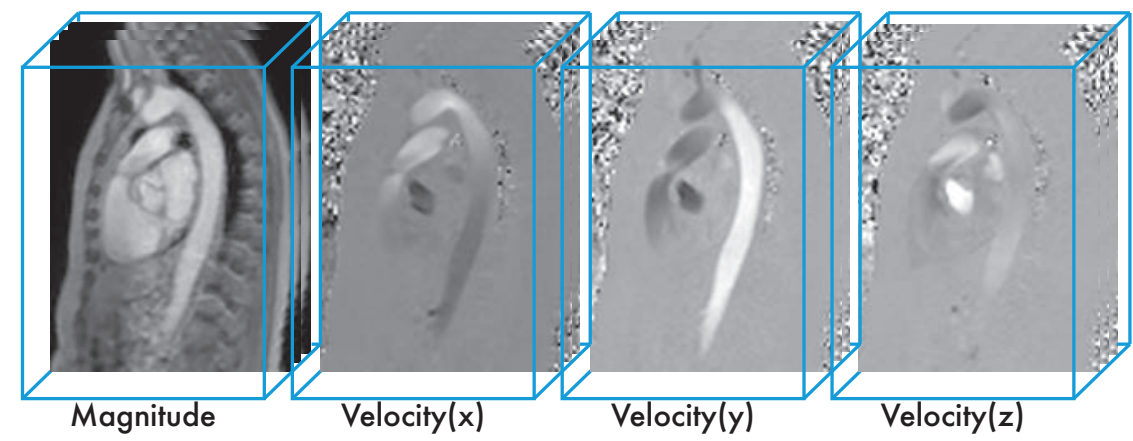

Figure 5: 4D Flow MRI dataset including anatomical information and velocities in three directions. Each box represents a four-dimensional volume.

4D Flow MRI can be defined as an extension of PC-MRI where flow-encoding has been performed in all three spatial directions relative to the three dimensions of space, also including the dimension of time along the cardiac cycle $[3,30,31]$. The resulting dataset is composed of four four-dimensional volumes, a magnitude, $M$, with anatomical information, and a velocity volume for each direction in space: 
$V_{x}, V_{y}$, and $V_{z}$. These volumes are isotropic or near-isotropic, with a typical spatial resolution of around $3 \mathrm{~mm}^{3}$ when imaging the whole heart and great vessels. A 4D Flow MRI dataset includes one cardiac cycle generated from a combination of multiple cardiac cycles collected over the course of the acquisition. The temporal resolution is usually $30-50 \mathrm{~ms}$, and the scan time is around 10 minutes for a volume covering the heart and great thoracic vessels. A 4D Flow MRI is illustrated in Figure 5.

The availability of this type of acquisition has added to the understanding of the physiology and cardiovascular flow patterns in health and disease [32], and has been shown to be valuable in clinical applications [33]. As the complete timeresolved three-directional three-dimensional velocity field is contained within the 4D Flow MRI, the blood flow can be analyzed using a large range of tools [5]. Quantitative measures, such as pressure, vorticity, kinetic energy, among others, can also be calculated and visualized based on 4D Flow MRI data [34-36]. Additionally, studies have also examined flow connectivity and distribution in the cardiovascular system over the cardiac cycle [16, 37].

\subsection{MR Angiography}

Magnetic Resonance Angiography (MRA) refers to a group of techniques based on magnetic resonance imaging created with the goal of improving the visualization of blood vessels. These techniques enable the generation of images that contain information about arteries and veins in order to evaluate them for stenoses, occlusions, aneurysms, or other abnormalities [38].

There are several different methods to obtain angiographic images using MR, among these, the ones most often used in the cardiovascular system and more closely related to this project are described more in detail in sections 4.5.1-4.5.3.

\subsubsection{Contrast-enhanced MRA (CE-MRA)}

When using CE-MRA, vascular images are produced using a contrast agent injected into the subject's blood system. This agent modifies the relaxation time of the regions in which it accumulates, which can be exploited by the acquisition sequence to increase the contrast of the blood in the resulting image [39]. An example of a CEMRA can be seen in Figure 6. CE-MRA imaging is independent of flow behavior, and data can be collected in a short amount of time with high signal-to-noise ratio. However, this technique requires good timing in order to acquire the image when the contrast agent is most concentrated in the regions of interest. Additionally, contrast agents are typically expensive, may be contraindicated in certain subjects, and accumulation of residual amounts of gadolinium-based contrasts in the brain has been reported [40]. 


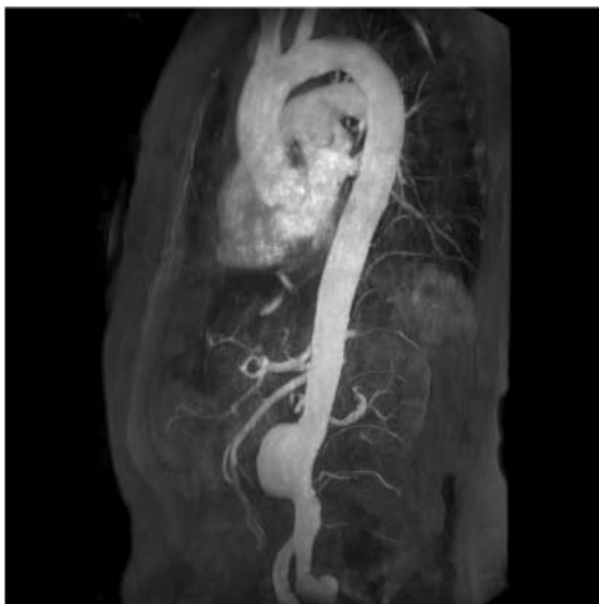

Figure 6: Maximum intensity projection of a contrast-enhanced MRA of the thoracic cardiovascular system.

\subsubsection{Phase-contrast MRA (PC-MRA)}

Phase-contrast MRA uses the same concepts as the previously described PC-MRI in order to generate angiographic images in which the flowing blood inside the vascular structures results in higher image intensities [41-43]. PC-MRA data are typically acquired without cardiac gating in a breath-hold. Consequently, the cardiac motion present during the acquisition is averaged in the resulting image, likely becoming difficult to perceive. As is the case with PC-MRI techniques, the resulting PC-MRA data allows for flow velocity quantification in the regions included.

\subsubsection{D Flow MRI generated angiography}

A 4D Flow MRI dataset can also be used to generate angiographic images similar to those obtained with PC-MRA, given the similarity of the underlying phase-contrast acquisition techniques present in both imaging methods. In this case, the magnitude and velocity images included in the 4D Flow MRI are combined, such as in equation 1 , in order to preserve higher intensities in areas where there is blood flow.

$$
\operatorname{PC}-\operatorname{MRA}(t)=\frac{1}{N} \sum_{t=t}^{N} M^{2}(t) * \sqrt{V_{x}^{2}(t)+V_{y}^{2}(t)+V_{z}^{2}(t)}
$$

In this equation, $t$ is a timeframe in the cardiac cycle, $N$ is the number of timeframes available in the dataset, $M$ is the magnitude component of the 4D Flow MRI, while $V_{x}, V_{y}$ and $V_{z}$ are the flow velocity components in the three spatial directions. A PCMRA generated from a 4D Flow MRI dataset using equation 1 can be seen in Figure 7. Different approaches of combining the 4D Flow MR data for the generation of angiographic images have been proposed and evaluated [44-46]. In most of 
these methods, the information over the cardiac cycle is averaged resulting in a three-dimensional image.

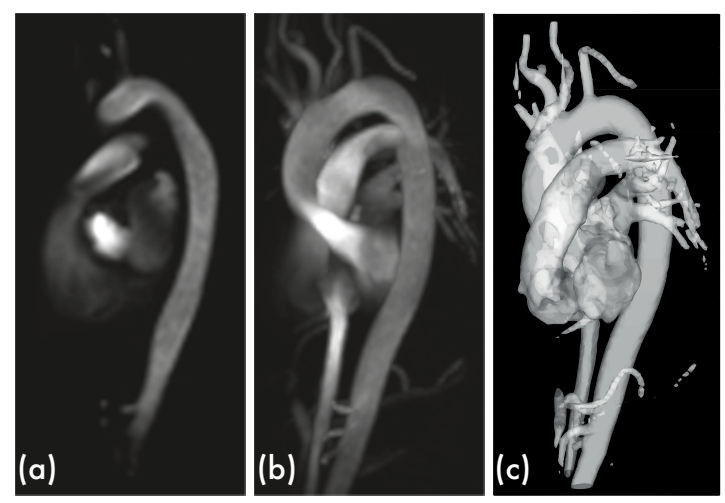

Figure 7: PC-MRA visualized as: (a) a coronal slice, (b) a maximum intensity projection, (c) an isosurface. 



\section{Chapter 5}

\section{Medical Image Analysis}

Medical image analysis consists of a series of techniques and procedures for the extraction of clinically useful information from the images acquired using a multitude of medical imaging methods such as radiography, ultrasound, magnetic resonance, computed tomography, among others. The goal of these techniques is to facilitate and improve the interpretation of medical images for diagnostic or therapeutic purposes.

Since their advent in the early twentieth century, medical imaging methods have greatly improved, both in respect to image quality, and also in relation to the time it takes to acquire and generate such images. In this context, as the amount of data generated by the modern health care system increases steadily, it creates a need for reliable processing techniques to assist in the interpretation of this vast source of information.

The subject of medical image analysis includes a broad range of techniques for a variety of objectives [47]. In this work, however, we will focus on the two methods most significant for this thesis: Image registration and image segmentation.

\subsection{Image Registration}

Image registration is the process of spatially aligning two or more images into a single frame of reference. It is used when the images have been acquired at different times, from different viewpoints, or by different sensors. Image registration is typically applied to solve problems such as scene reconstruction, object detection and recognition, motion analysis and compensation, change detection, image fusion, or object tracking. This section offers a concise introduction to the extensive topic of image registration; for a more detailed explanation, please refer to [48-50].

In general terms, the problem of image registration can be defined as follows: Given a fixed or reference image, $I_{F}(x)$, and a moving or template image $I_{M}(x)$, find a displacement, $u$, such that the transformed moving image, $I_{M}(x+u(x))$ is spatially aligned to the fixed image, $I_{F}(x)$. The expression $x+u(x)$ can also be defined as a transformation, $T=x+u(x)$, that maps the moving image to the fixed image. A straightforward registration method typically consists of the following components:

- Distance measure: Also referred to as similarity measure, defines the quality 
of the spatial alignment calculated by the registration method. The registration is finished when the transformed moving image $I_{M}(x+u(x))$ is sufficiently similar to the fixed image $I_{F}(x)$. A few well-known distance measures typically used in image registration are the sum of squared differences, sum of absolute differences, mutual information, and cross-correlation [51].

- Transformation model: Determines the set of transformations that are allowed during the registration process in order to align the images. Transformation models used in image registration can be divided into rigid and nonrigid. Rigid transformations only include rotation and translation, while nonrigid models allow for more complex transformations, such as skewing and shearing, and can in term be subdivided into parametric and non-parametric transformations.

- Optimization method: Indicates the technique used to calculate the optimal transformation, $\hat{T}$, for which the moving image is sufficiently similar to the fixed image. With the goal of image registration, standard numerical optimization methods, such as gradient descent, Quasi-Newton, conjugate gradient, among others, are usually employed [52].

Once all the components have been selected, the registration method can be defined as an optimization problem where the similarity measure must be maximized until an optimal transformation $\hat{T}$ is obtained:

$$
\begin{gathered}
\hat{T}=\underset{T}{\arg \min } C\left(T ; I_{F}, I_{M}\right), \\
C\left(T ; I_{F}, I_{M}\right)=D\left(T ; I_{F}, I_{M}\right)+\alpha R(T)
\end{gathered}
$$

where $C$ is the cost function to minimize, $D$ is the distance measure, $\alpha$ weighs similarity of the images versus the smoothness of the displacement field, and $R$ is a regularization term that determines the smoothness of the field, typically included to constraint $T$ to be continuous and physically plausible.

A great number of registration methods have been implemented by extending and improving this relatively simple framework. These techniques have been evaluated in medical imaging in general $[53,54]$, and specifically in cardiovascular imaging $[55,56]$. In cardiovascular image analysis, registration has been used for a variety of tasks, such as comparison of images acquired at different times or by different imaging modalities [57], motion artifact correction [58, 59], motion and strain quantification $[60,61]$, segmentation $[62,63]$, and generation of statistical shape models $[64,65]$.

The main challenge of registration in this field of study is its high requirement for computational resources, which can make image registration a time-consuming process. Additionally, deformations applied on medical images can result in morphological changes in the physiological structures of interest, which must be carefully considered when using these images for diagnostic assessment. 


\subsubsection{Registration Considerations for this Thesis}

Registration is an extensive topic of research in the field of image analysis. Consequently, there is a considerable number of implementations and parameters to be selected within each possible implementation in order to address the specific registration problem we attempt to solve.

In this work, registration was used to align MR images acquired with different techniques and for a variety of purposes, such as motion correction, time-resolving, and segmentation. Therefore, we experimented with several registration techniques previously implemented, and explored the best parameters to apply for each particular question. This section aims to give a theoretical introduction to the concepts that we found useful during this project regarding image registration.

\section{Distance Measures}

A wide variety of methods for the quantification of the degree of similarity between two images have been proposed and evaluated for medical image analysis [51, 66, 67]. Amongst them, the ones used at different stages of this thesis were:

- Sum of Squared Differences (SSD): This is a common, easy to use method. However, it assumes that the intensities in the images are directly comparable, which might not be the case regarding images acquired with different modalities. The SSD between two images, $I_{F}$ and $I_{M}$, can be calculated as:

$$
\operatorname{SSD}\left(I_{F}, I_{M}\right)=\int_{\Omega}\left(I_{M}(x+u(x))-I_{F}(x)\right)^{2} d x
$$

- Normalized Cross Correlation (NCC): Estimates the strength of the relationship between two signals, in this case represented by the compared images. Given a fixed and a moving image, $I_{F}$ and $I_{M}$, respectively, and $T$, a spatial transformation mapping the moving to the fixed image, the NCC can be calculated as follows:

$$
\operatorname{NCC}\left(I_{F}, I_{M}\right)=\frac{\sum_{x_{i} \in \Omega_{F}}\left(I_{F}\left(x_{i}\right)-\overline{I_{F}}\right)\left(I_{M}\left(T\left(x_{i}\right)\right)-\overline{I_{M}}\right)}{\sqrt{\sum_{x_{i} \in \Omega_{F}}\left(I_{F}\left(x_{i}\right)-\overline{I_{F}}\right)^{2} \sum_{x_{i} \in \Omega_{F}}\left(I_{M}\left(T\left(x_{i}\right)\right)-\overline{I_{M}}\right)^{2}}},
$$

where $\overline{I_{F}}$ and $\overline{I_{M}}$, are the average values of the fixed and moving images, calculated as:

$$
\begin{aligned}
& \overline{I_{F}}=\frac{1}{\left|\Omega_{F}\right|} \sum_{x_{i} \in \Omega_{F}} I_{F}\left(x_{i}\right) \text {, and } \\
& \overline{I_{M}}=\frac{1}{\left|\Omega_{F}\right|} \sum_{x_{i} \in \Omega_{F}} I_{M}\left(T\left(x_{i}\right)\right)
\end{aligned}
$$


$\Omega$ represents the domain of the image, and $\left|\Omega_{r}\right|$ the number of pixels in the image.

- Normalized Mutual Information (NMI): Mutual information is a measure of the mutual dependence between the images $[68,69]$. It is a similarity measure typically used for multi-modality image registration, since it does not depend directly on the intensities of the images, but on the statistical relationship between these intensities. In terms of entropy, $H$, which serves to evaluate the degree of unpredictability of a state, the NMI can be calculated as:

$$
\operatorname{NMI}\left(I_{F}, I_{M}\right)=\frac{\left(H\left(I_{F}\right)+H\left(I_{M}\right)\right)}{H\left(I_{F}, I_{M}\right)}
$$

Additionally, as defined in [70], the NMI can be calculated using B-spline Parzen windows as:

$$
\operatorname{NMI}\left(I_{F}, I_{M}\right)=\frac{\sum_{m \in L_{M}} \sum_{f \in L_{F}} p(f, m) \log _{2}\left(p_{F}(f) p_{M}(m)\right)}{\sum_{m \in L_{M}} \sum_{f \in L_{F}} p(f, m) \log _{2} p(f, m)}
$$

where $L_{F}$ and $L_{M}$ are sets of regularly spaced intensity bin centers, $p$ is the discrete joint probability function, and $p_{F}$ and $p_{M}$ are the marginal discrete probabilities of the fixed and moving images, respectively. In both equations, $I_{F}$ and $I_{M}$ represent the fixed and moving images.

- Local Phase Difference: Local phase is a concept derived from signal processing with the aim of measuring local shape [71]. It is invariant to local variations in the signal intensity, which is an advantage when used for image registration. This measure is the basis for the Morphon registration algorithm [72], described in more detail at the end of this section.

The phase is defined as a one-dimensional measure, however, for a multidimensional image, it can be calculated using a set of quadrature filters, $f_{k}$, with $K$ different orientations, $\hat{n}_{k}$. Given a fixed and moving image, $I_{F}$ and $I_{M}$, respectively, their phase-difference for orientation $k, \Delta \varphi_{k}$, can be calculated as the argument of the complex conjugate product of the quadrature filter responses:

$$
\begin{gathered}
\Delta \varphi_{k}=\arg \left(Q_{k}\right) \\
Q_{k}=q_{F, k} q_{M, k}^{*}
\end{gathered}
$$

where $q_{k}$ denote the filter responses from the quadrature filter:

$$
\begin{gathered}
q_{F, k}=I_{F} * f_{k} \\
q_{M, k}=I_{M} * f_{k}
\end{gathered}
$$


Additionally, local phases allow for the calculation of another useful term, the local structure tensor, used to describe the orientation of signal variation [73]. It can be calculated as a summation of the quadrature filter output magnitudes as follows:

$$
T=\sum_{k}\left\|q_{k}\right\|\left(\hat{n}_{k} \hat{n}_{k}^{T}-\alpha I\right)
$$

where $\alpha=\frac{1}{m-1}, m$ being the dimensionality of $T$, and $I$ is the identity tensor.

\section{Transformation Models}

As mentioned previously, the transformation models used during image registration can be divided into parametric and non-parametric models, both of which where used in this work.

In a parametric model, the transformation is defined as a function of independent parameters. Examples of parametric transformations typically used in image registration include rigid, affine, radial basis functions, and B-splines. When using a low dimensional set of parameters, regularization of the resulting transformation field is implicitly included in these transformations.

Non-parametric models estimate the transformation as an unknown function without explicit parameterization. This represents an ill-posed problem since its solution is not unique, and consequently, requires a regularization term to be applied to the transformation. Regularization of the estimated transformation field is used to guarantee certain characteristics in the solution such as smoothness, sparsity, or rigidity, while at the same time avoiding cracks, foldings, or undesirable deformations.

\section{The Morphon Algorithm}

In this work, non-rigid registration was applied using a non-parametric framework based on the Morphon algorithm [74]. The Morphon was first described in [72] and has been used previously for several medical image processing tasks, such as atlas-based segmentation [75-77] and anatomical atlas generation [78, 79].

The Morphon algorithm makes use of local phase differences as a measure of local structure for the estimation of displacement fields between the input images. Consequently, the algorithm is less sensitive to variations in the image intensities when compared to other methods that rely on these intensities directly.

A displacement field between the moving and the fixed images, $I_{M}$ and $I_{F}$, is accumulated from a number of iterations using multiple scales that progress from coarser to finer. In each iteration, an update to the current displacement field, $\delta u$, is calculated as the solution to the following least squares problem:

$$
\delta u=\min _{d} \sum_{k} \frac{1}{2}\left\|c_{k} T\left(d_{k} \hat{n}_{k}-d\right)\right\|^{2}
$$

where $d$ is the estimated displacement, $T$ is the local structure tensor of $I_{M}$ and $I_{F}$, calculated as the average tensor of the two images (see equation 14), $\hat{n}_{k}$ is the 
direction of filter $k, d_{k}$ is the displacement estimate in direction $k$, and $c_{k}$ is a certainty term used to reduce the effect of noise in the local phase corresponding to direction $k$, and it is calculated as:

$$
c_{k}=\sqrt{Q_{k}} \cos ^{2}\left(\frac{\Delta \varphi_{k}}{2}\right)
$$

Equations 10 and 11 show how to calculate the local phase differences $\Delta \varphi_{k}$ and $Q_{k}$.

Additionally, a certainty map $\delta c$, related to $\delta u$ can be defined as:

$$
\delta c=\sum_{k} c_{k}
$$

The total displacement field for each iteration is the result of accumulating the displacement field, $u$, calculated in the previous iteration and the update field $\delta u$. This is done using the following formula:

$$
u=\frac{c u+\delta c(u+\delta u)}{c+\delta c}
$$

where $c$ is the certainty map related to the displacement field $u$, calculated using $\delta c$ as:

$$
c=\frac{c^{2}+\delta c(c+\delta c)}{c+\delta c}
$$

Once the displacement field has been calculated for the current iteration, the next step consists of regularization of the field in order to make it smooth and ensure a physically plausible transformation. This can be accomplished in a number of ways; however, during this work we focused on the following regularization methods:

- Elastic regularization: Calculated using the elastic potential of the displacement field $u$ [80], defined as:

$$
R(u)=\frac{1}{2} \int_{\Omega} \mu\langle\nabla u, \nabla u\rangle+(\lambda+\mu)(\nabla \cdot u)^{2} d x
$$

where $\lambda$ and $\mu$ are the Lamé parameters that define material properties of the field.

- Fluid regularization: Uses the characteristics of a viscous fluid to model the displacement field [81]. It is calculated using the elastic potential of the velocity of the displacement field $u$ :

$$
R(v)=\frac{1}{2} \int_{\Omega} \mu\langle\nabla v, \nabla v\rangle+(\lambda+\mu)(\nabla \cdot v)^{2} d x
$$

where $v$ is a velocity field defined as $v=\partial_{t} u+v^{T} \nabla u, t$ is an artificial time introduced for this calculation. 


\subsection{Image Segmentation}

The goal of segmentation is to divide the image into sections that represent objects or areas of the real world. Different characteristics of the images such as noise, artifacts, or low contrast of the structures of interest can make segmentation quite a challenging process. Moreover, even when performed by a trained expert, manual segmentation can be slow, tedious, and difficult to reproduce. Therefore, automatic segmentation methods have been a focus of research since the beginnings of image analysis [82, 83].

As is the case with the most useful image analysis techniques, extensive research has been dedicated to develop and expand a great variety of segmentation approaches. Some of the most commonly used techniques in medical image analysis are based on thresholding, edge-detection, region-growing or merging, clustering, pattern recognition, or atlases, to name a few [84, 85].

Within medical imaging, the typical areas segmented are different tissues, organs, or pathologies. Segmentation is one of the most important challenges in medical image analysis, since it is usually necessary to identify any anatomically relevant structures in the image before useful information can be extracted [86, 87]. More specifically, in cardiovascular imaging, segmentation is performed with the goal of assessing myocardial motion, ventricular stroke volume or shape, assessment of vessel geometry, flow analysis, among others [88-90].

Atlas-based segmentation is one among the several segmentation methods available that has lead to good results in medical imaging [91, 92]. The following section expands further on this particular method, which is the basis for the segmentation techniques proposed in this thesis.

\subsubsection{Atlas and Multi-atlas Segmentation}

In the context of medical image analysis, an atlas, sometimes also called a template, is an image that incorporates locations and shapes of anatomical structures, and the spatial relationships between them. An atlas can be generated by incorporating information from multiple segmented images, or, on the other hand, when using multi-atlas segmentation, several independent atlases can be combined into one final segmentation [93].

Atlas and multi-atlas segmentation benefit from the non-trivial medical expertise included in the atlases. This is particularly important for medical imaging, where knowledge of the anatomy depicted in the images is essential for a successful segmentation.

Atlas-based segmentation permits locating and labeling specific areas in the cardiovascular image by registering the atlas to an unsegmented image. Using registration, a deformation is calculated in order to account for morphological differences between the atlas and the image to be segmented. The deformed atlas will then indicate the location of the areas it represents in the new image [94, Chapter 11]. The use of multiple atlases strengthens the segmentation method by improving its abil- 
ity to handle anatomical variation between subjects, consequently achieving better segmentation accuracy [92].

In the past decade, multi-atlas segmentation methods have been successfully tested on several different types of medical MR images, such as brain MRI [95, 96], prostate MRI [97], cardiac MRI [98, 99], whole body MRI [100], breast tissue MRI [101], knee MRI [102], among others.

\section{Label Fusion in Multi-Atlas Segmentation}

In multi-atlas segmentation, the registered atlases represent a set of possible solutions to the problem from which a final segmentation can be extracted. Several studies have proposed different techniques to merge or fuse all these solutions into one final labeling [97, 103-109].

When implementing multi-atlas segmentation in this work, label fusion was accomplished using the simultaneous truth and performance level estimation (STAPLE) algorithm [103], which has been used previously for consensus ground truth calculation for cardiac MR segmentation methods [110, 111].

STAPLE uses expectation-maximization (EM) [112] to calculate a probabilistic estimate of the true segmentation based on the group of segmentations provided by the registered atlases. EM is a widely applicable approach for computing maximum likelihood estimates from incomplete data. When applied in multi-atlas segmentation, the incomplete data is the collection of expert decisions, $D$, in this case represented by the registered atlases, while the complete data also includes the true segmentation $T$.

Let $D$ be an $N \times R$ matrix describing the binary decisions made by $R$ experts for each of the $N$ voxels of the image. $T$ is the hidden true segmentation of $N$ elements to be estimated by the algorithm. The quality of each expert segmentation, $r$, is represented by the performance level parameters sensitivity and specificity, $p$ and $q$, respectively, at each voxel $i$ :

$$
\begin{aligned}
& p_{r}=P\left(D_{i r}=1 \mid T_{i}=1\right) \\
& q_{r}=P\left(D_{i r}=0 \mid T_{i}=0\right)
\end{aligned}
$$

$p_{r}$ denotes the likelihood that expert $r$ correctly identifies a voxel inside the true segmentation, while $q_{r}$ is the likelihood that the expert correctly identifies a background voxel as such. STAPLE estimates the performance level parameters of the experts which maximize the complete data log likelihood function:

$$
(\hat{p}, \hat{q})=\arg \max _{p, q} \ln f(D, T \mid p, q)
$$

where $f(D, T \mid p, q)$ is the probability mass function of the complete data $(D, T)$. At iteration $k$, the performance parameters that maximize the conditional expectation of the log likelihood function can be estimated as:

$$
\left(p^{(k)}, q^{(k)}\right)=\arg \max _{p, q} E\left[\ln (f(D, T \mid p, q) f(T)) \mid D, p^{(k-1)}, q^{(k-1)}\right]
$$


When used in STAPLE, the EM algorithm works by iterating two steps until convergence:

1. The first step (E-step, expectation) estimates the conditional probability of the true segmentation, $W$, given the registered atlas labels and the estimates of sensitivity and specificity, $p^{(k-1)}$ and $p^{(k-1)}$, calculated in the previous iteration $(k-1)$ :

$$
W_{i}^{(k-1)} \equiv f\left(T=1 \mid D_{i}, p^{(k-1)}, q^{(k-1)}\right)
$$

For each voxel in the image, $W_{i}$ denotes the likelihood that voxel $i$ corresponds to 1 in the ground truth segmentation, i.e., $W_{i}=P\left(T_{i}=1\right)$.

2. In the second step (M-step, maximization) the new performance level estimates, $p^{(k)}$ and $q^{(k)}$, are updated using $W^{(k-1)}$ as:

$$
\begin{gathered}
p_{j}^{(k)}=\frac{\sum_{i: D_{i j}=1} W_{i}^{(k-1)}}{\sum_{i} W_{i}^{(k-1)}} \\
q_{j}^{(k)}=\frac{\sum_{i: D_{i j}=0}\left(1-W_{i}^{(k-1)}\right)}{\sum_{i}\left(1-W_{i}^{(k-1)}\right)}
\end{gathered}
$$

\subsubsection{Evaluating Segmentation}

A broad range of metrics have been proposed in order to measure the quality of a segmentation [113]. This is a very important step in the evaluation of automatic or semi-automatic segmentation techniques. In medical imaging, it typically consists of a comparison between the generated segmentation and the ground truth, which is generally obtained using the clinical gold standard, considered to be the most accurate segmentation possible.

Segmentations generated or corrected in this work were evaluated using the following metrics:

- Dice Similarity Coefficient (DSC): First presented in [114], is one of the most commonly used metrics for image segmentation evaluation. It is a measure of overlap between the input volumes, calculated as:

$$
\mathrm{DSC}=\frac{2|A \cap B|}{|A|+|B|}
$$

where $A$ and $B$ are the segmentations we wish to compare. The operator $|A|$ is defined as the number of elements (pixels or voxels) included in the segmentation. The resulting coefficient is a number in the range $[0,1]$, where zero represents no overlap while one signifies complete overlap. 
- Hausdorff Distance (HD): It is defined between two sets of points as the maximum of all the distances from a point in one set to its closest point in the other set [115]. Given two finite sets of points $A$ and $B$, the Hausdorff distance is given by:

$$
\mathrm{HD}=\max (h(A, B), h(A, B))
$$

where $h(A, B)$ is the directed Hausdorff distance, calculated as:

$$
h(A, B)=\max _{a \in A} \min _{b \in B}|| a-b \|
$$

$\|a-b\|$ is a norm, such as the Euclidean distance.

In addition to the geometric measures, the proposed methods were also evaluated using the following morphological and flow related criteria:

- Cardiac volume: Segmentations of the cardiac chambers at representative timeframes of the cardiac cycle, such as end-systole and end-diastole, are typically done in the clinic in order to assess cardiac function. Volume comparison between the segmentations at these timeframes has been previously used as a measure of quality in cardiac imaging. This is particularly useful when evaluating segmentations generated using images obtained with different imaging acquisition methods, since cardiac volume should remain the same for the different images.

- Stroke Volume (SV): Volume of blood pumped by a cardiac ventricle in a heartbeat, used to measure cardiac efficiency. The stroke volume for the left and right ventricles should be equal in a heart without shunts, which can be used a marker for cardiac disease, and additionally, as an evaluation measure for segmentation. It can be calculated using equation (32).

$$
\mathrm{SV}=\mathrm{EDV}-\mathrm{ESV}
$$

where EDV (end-systolic volume) is the volume of blood in the cardiac chamber at end-diastole, and ESV (end-systolic volume) is the volume of blood in the chamber at end-systole.

- Ejection Fraction (EF): Fraction of blood expelled from a cardiac ventricle in a heartbeat, calculated using equation (33). It is also a measure of cardiac efficiency.

$$
\mathrm{EF}=\frac{\mathrm{SV}}{\mathrm{EDV}} \times 100
$$

- Flow Volume $(\mathbf{Q})$ : Measure of the volume of fluid that passes through an area in a specific amount of time. Within the field of cardiology, it is often used to account for the volume of blood that travels through a vessel in one heartbeat. It is frequently calculated at a plane located in the proximal ascending aorta 
(systemic flow volume, $Q_{s}$ ), or at a plane located in the pulmonary trunk (pulmonary flow volume, $Q_{p}$ ). The flow volume can be calculated as follows:

$$
\begin{gathered}
\mathrm{Q}=\int_{t} \mathrm{v} d t \\
\mathrm{~V}=v \cdot A
\end{gathered}
$$

where $v$ is the through-plane flow velocity, and $A$ is the cross-sectional vector area of the vessel.

With the goal of segmentation evaluation, $Q_{s}$ can be compared to the left ventricular stroke volume, while $Q_{p}$ should correspond to the right ventricular stroke volume. 



\section{Chapter 6}

\section{D Flow MRI Assessment}

Besides having long acquisition times, application of 4D Flow MRI has been hindered by the complexity of the analysis of these comprehensive datasets [3]. Analysis of 4D Flow MRI data can be extremely time consuming, especially during the segmentation stage which is a requirement for most assessment methods. Additionally, in 4D Flow MR images, the contrast between the myocardium and the blood is typically not very high, resulting in the need to include other MR acquisitions during the analysis method.

This chapter provides a description of the procedures frequently followed in order to generate clinically useful information from 4D Flow MRI datasets.

\subsection{Vessel Segmentation}

Several studies have combined the 4D Flow MRI magnitude and velocity information in order to obtain an angiographic visualization of the thoracic blood vessels [45], see section 4.5 .3 for a brief description of these techniques. The resulting angiograms have then been employed to segment the main vessels contained in the 4D Flow MRI dataset using a variety of approaches: level sets [116-118], watershed transformation [46], graph cuts [119], among others. These techniques typically result in a three-dimensional segmentation of the vessels, ignoring any vessel movement that occurs during the cardiac cycle, which might be sufficient for the assessment of certain hemodynamic parameters that are not affected by motion.

Previous studies have generally focused on three-dimensional segmentation due to its relative simplicity and shorter time requirements with respect to fourdimensional segmentation methods; however, some semi-automatic attempts have been made [120]. Four-dimensional segmentations can be useful for calculations that must be performed near the vessel walls or for a more detailed visualization of blood flow throughout the cardiac cycle.

\subsection{Cardiac Segmentation}

Current methods for 4D Flow MRI cardiac segmentation incorporate the usage of separately acquired b-SSFP MRI in order to segment the cardiac ventricles and atria 
[24]. These images have higher in-plane resolution and better contrast between the blood and the myocardium than 4D Flow MR images, and are consequently frequently used to locate and segment the cardiac chambers. However, the use of b-SSFP MR images also introduce some extra challenges when attempting to use the segmentations on 4D Flow MRI data:

- When acquired for cardiac assessment, b-SSFP MR images usually include a short-axis stack that covers the cardiac anatomy. As discussed in section 4.3, b-SSFP MR images are acquired during a series of breath-holds. Typically, only one or two slices of the stack are acquired per breath-hold, and about one breath-hold is taken per minute. In order to decrease the acquisition time, the short-axis stack is made to cover the cardiac ventricles (from the apex to the ventricular outflow tract), and exclude the complete cardiac atria and major thoracic vessels, which are frequently contained in the 4D Flow MRI. Part of the cardiac atria can be observed in the b-SSFP long-axis images that are usually included in the imaging sequence. However, these images only offer a partial depiction of the atrial chambers and are not enough to discern their precise shape.

- The images included in the b-SSFP MR short-axis stack are typically acquired with a relatively large slice thickness (between 7 and 10mm) in order to increase the signal-to-noise ratio in the image plane. The resulting lower resolution in the direction perpendicular to the image plane is sufficient for myocardial motion assessment and calculation of cardiac stroke volume and myocardial volume. However, the large slice thickness can affect the quality of the segmentation when superimposed on the isotropic or near-isotropic 4D Flow MR images.

- The different slices of the short-axis stack are acquired over the course of several breath-holds. Therefore, misalignments between the slices of the stack are quite common when the position of the subject's diaphragm changes between breath-holds $[25,121]$.

Advanced methods for semi-automatic or automatic ventricle segmentation focusing on b-SSFP MRI have been previously presented, using techniques such as deformable models [122], active shape and appearance models [123], atlas-guided segmentation [124], among others. A review of segmentation methods in short- and long-axis cardiac MR images can be found in [90, 125]. These techniques have reduced the processing time needed to analyze b-SSFP MR images, but have not been made to be used directly on 4D Flow MRI. Moreover, the 4D Flow MR images include more regions of interest, such as the atria and thoracic vessels, which could also be segmented and analyzed. 


\subsection{Visualization}

Many approaches for visualization of intracardiac blood flow have been published over the past decades [2,126-128]. Visualization can be performed using cut planes, vector maps, maximum intensity projections, isosurfaces, volume renderings, and particle traces, to name a few. The type of visualization used must be based on the particular goal of the study, moreover, choosing the right way of visualizing the data can be essential in understanding the processes that take place within the complex 4D Flow MRI datasets.

Particle traces, i.e. streamlines and pathlines, have become especially popular for intuitive visualization of time-resolved three-dimensional blood flow in the heart and vessels. Streamlines are lines parallel to the velocity vector field at an instant in time, they are useful for representing instantaneous flow characteristics. On the other hand, pathlines are calculated by integrating the vector field over time; they follow the path that an imaginary particle, or a tiny drop of dye, would take over time. Pathlines are suitable for inspecting flow features through time.

Isosurfaces, maximum intensity projections, and volume renderings have been used for visualization of anatomical landmarks, or to depict hemodynamic parameters that have a structure, such as vortex rings or turbulence $[30,129,130]$.

\subsection{Hemodynamic Analysis}

As the complete time-resolved three-directional three-dimensional velocity field is contained within the 4D Flow MRI, its data can be analyzed using a large range of tools [5]. Using these velocity fields, hemodynamic markers such as flow volume, pressure, vorticity, kinetic energy, pulse wave velocity, turbulence, helicity, wall shear stress, flow connectivity, among others, can also be calculated and visualized [16, 34-37].

Many of the studies regarding hemodynamic assessment of cardiovascular diseases consist of comparisons between patients and healthy volunteers in order to derive markers of disease $[19,35,129,131,132]$. There has also been some effort in recent years to create tools to generate group-wise average quantification and visualization of hemodynamic parameters $[12,13]$. The underlying idea is to create statistical models of the behavior of certain parameters under disease to aid in clinical understanding and diagnosis of different conditions.

A subset of the currently existing hemodynamic parameters were calculated during the course of this thesis in order to evaluate the proposed techniques, and to compare different datasets or groups of datasets to each other. The following is a list of these parameters and their definitions:

- Flow Volume (Q): Described previously in section 5.2.2, the flow volume can be calculated using equation (34).

The flow volumes in the proximal ascending aorta and pulmonary trunk, $Q_{S}$ and $Q_{p}$, respectively, are commonly used to calculate the pulmonary-systemic 
flow ratio $\left(Q_{p} / Q_{s}\right)$, which can be used in a similar way to the comparison of stroke volumes between the left and right ventricle. In a healthy heart, without any septal defects, the outputs of the left and right ventricles should be the same, i.e., the pulmonary-systemic ratio should be close to one.

- Kinetic Energy (KE): Measure of the energy of an object generated by its movement. In the context of cardiovascular hemodynamic analysis, alterations in the $\mathrm{KE}$ of the cardiovascular flow can be a consequence of the loss of energy in the system, which can be caused by a decrease in cardiac efficiency. Consequently, KE has been previously used as a marker of disease in both the heart and vessels $[19,133,134]$. Voxel-wise KE of the blood can be computed using equation (36).

$$
\mathrm{KE}=\frac{1}{2} m v^{2}
$$

where $m$ represents the mass, and $v$ the blood's speed. Using the formula to calculate density $(\rho), m$ can be calculated as:

$$
m=\rho d V
$$

where $\rho$ is the blood's density, with a known average value of $1060 \mathrm{~kg} / \mathrm{m}^{3}$, and $d V$ is the volume per voxel.

- Helicity (H): Denotes the extent of helical patterns within the flow, observed when a fluid rotates around an axis that follows the main direction of flow. It is a pseudo-scalar; consequently, it changes sign depending on the direction of the helical patterns. It has been previously used in the analysis of the typically helical aortic flow $[135,136]$. The equation to calculate $\mathrm{H}$ in a volume $\mathrm{V}$ is:

$$
H=\int_{\mathrm{V}} \vec{u} \cdot \omega d V
$$

where $\vec{u}$ is the velocity vector, and $\omega$ is the vorticity or curl of the velocity vector field, defined as:

$$
\omega=\nabla \times \vec{u}=\left(\frac{\partial v_{z}}{\partial y}-\frac{\partial v_{y}}{\partial z}, \frac{\partial v_{x}}{\partial z}-\frac{\partial v_{z}}{\partial x}, \frac{\partial v_{y}}{\partial x}-\frac{\partial v_{x}}{\partial y}\right)
$$

Additionally, the density of the helicity $\left(H_{d}\right)$ can be calculated as the dot product between the velocity vector and the vorticity:

$$
H_{d}=\vec{u} \cdot \omega
$$




\section{Chapter 7}

\section{Proposed Techniques}

The following sections contain a detailed description of the methods proposed in the papers included in this thesis. These techniques were developed with the aim of increasing the clinical utility of 4D Flow MRI, and as such, they focus on improving, facilitating, and expediting some of the steps in the current standard of 4D Flow MRI assessment.

\subsection{Automatic Segmentation Correction}

As discussed in section 6.2, the current standard for cardiac image segmentation in 4D Flow MRI uses short- and long-axis b-SSFP MR images of the cardiac anatomy due to their comparatively higher myocardial contrast and in-plane resolution. Frequently, the cardiac chambers are delineated manually by an expert or using semiautomatic methods. In addition to the challenges already involved when manual operations are required, the specific characteristics of the b-SSFP MR images also incorporate some extra challenges to the utility of the resulting segmentations for 4D Flow MRI analysis. Among these challenges, the issue of misalignments between slices caused by differences in the thorax's location during the distinct breath-holds required for the b-SSFP MRI acquisition can particularly hinder flow assessment when using a segmentation created from a misaligned short-axis stack on the 4D Flow MRI [19, 132].

In Paper I, “Improving Left Ventricular Segmentation in Four-Dimensional Flow MRI Using Intramodality Image Registration for Cardiac Blood Flow Analysis", we proposed a method to automatically align the slices of the b-SSFP short-axis stack to the 4D Flow MRI using rigid registration. The technique consists of the following steps:

1. A region of interest (ROI) is automatically defined to contain the heart and its closely surrounding areas in order to exclude other areas of the anatomy that might negatively affect the registration. This is an optional step and may be ignored if desired.

2. For each slice of the short-axis stack of the b-SSFP MRI: 
(a) Calculate the intersection between the slice and the 4D Flow MRI as an interpolation of the 4D Flow MRI magnitude image at each voxel of the b-SSFP MR slice.

(b) Use rigid registration to calculate a transformation, $\hat{T}$, between the intersection generated in the previous step and the b-SSFP MR slice.

(c) Apply the transformation $\hat{T}$ to the b-SSFP MR slice.

A result of the proposed method can be seen in Figure 8, and an example of the effect of correction on the flow pathlines generated using the 4D Flow MRI can be seen in Figure 9.
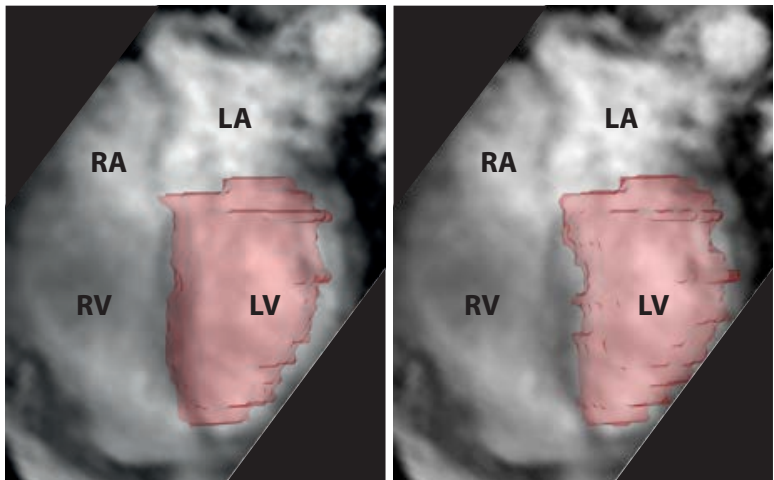

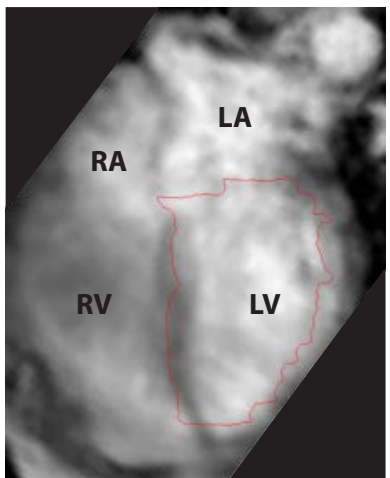

Uncorrected

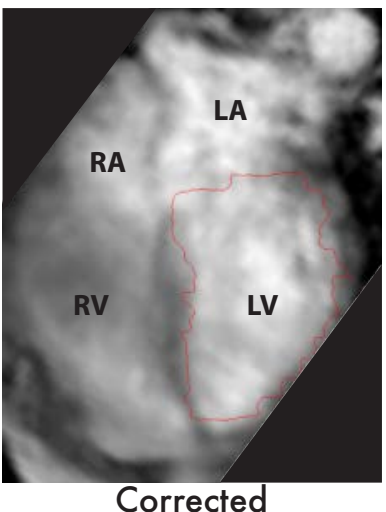

Corrected

Figure 8: Uncorrected versus corrected segmentations for one dataset. The left ventricular segmentation is superimposed over a four-chamber view of the heart using an isosurface (top) and the isosurface boundary (bottom). 

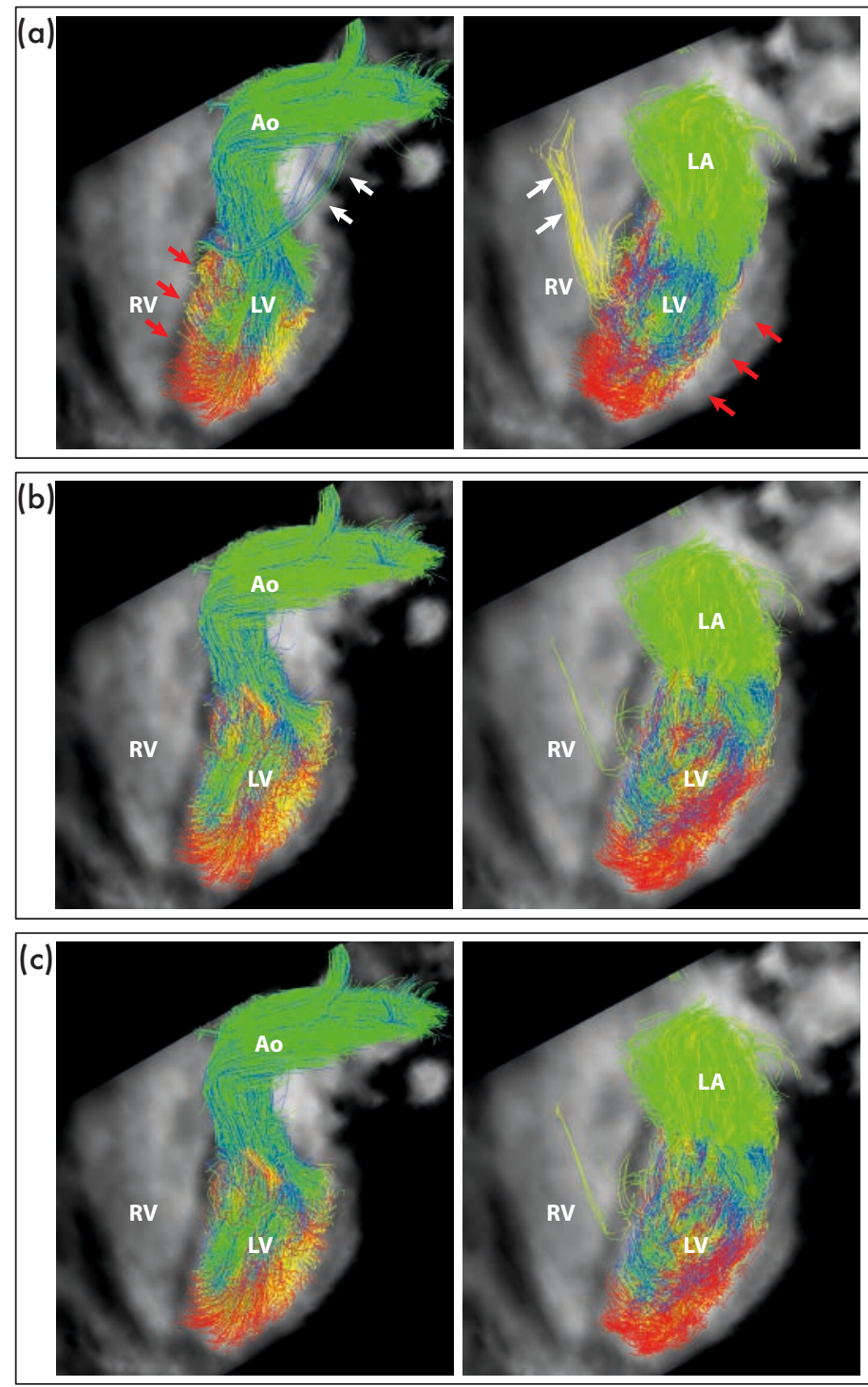

Figure 9: A representative example of the effect of segmentation correction on the flow pathlines generated using 4D Flow MRI. (a) Uncorrected, (b) Expert corrected, (c) Automatically corrected. The leftmost figures correspond to the outflow pathlines, and the rightmost to the inflow pathlines. Visible errors caused by misalignment of the short-axis images are depicted with red arrows, while errors affecting the generated pathlines are shown with white arrows. The pathline colors were defined in a previous study with the goal of ventricular flow analysis [16]. 
The rigid registration included in the method was evaluated for two different similarity measures: normalized cross-correlation (NCC) [137], and normalized mutual information (NMI) [70], with NMI resulting in the best outcomes during the evaluation. The implementation of this method employed an open-source registration framework, Elastix. [138].

The method was tested in 20 subjects, 10 healthy volunteers and 10 patients with ischemic cardiomyopathy or idiopathic dilated cardiomyopathy. To evaluate the technique, we calculated the geometric accuracy of the automatic correction by comparison with manual correction performed by an expert. Two well-known methods for the calculation of spatial correspondence were used: Dice Similarity Coefficient (DSC), and Hausdorff distance (HD). Additionally, we evaluated the effect of the correction in the volume of flow calculated using pathlines going in and out of the left ventricle [16].

The correction method resulted in reduced differences between the expert corrected and the automatically corrected segmentations when compared to the uncorrected labels for all the evaluation methods included in the study. See Figure 10 for the geometric accuracy results, and Figure 11 for the flow analysis comparison between uncorrected and corrected segmentations.
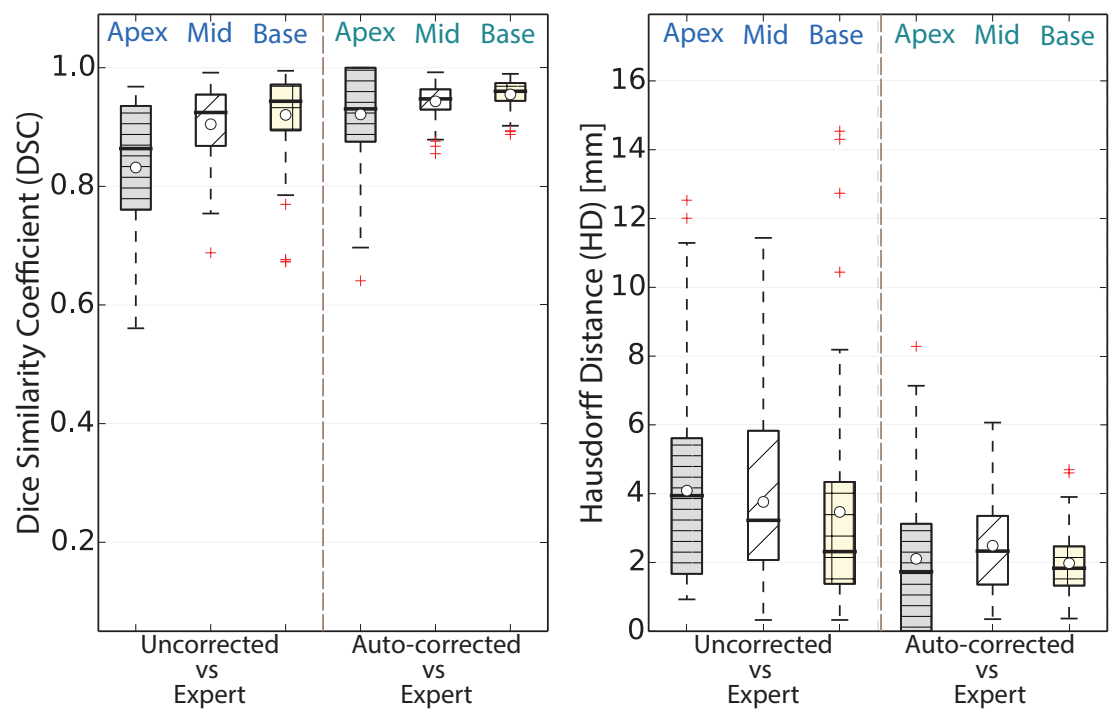

Figure 10: Geometric accuracy results for three different sections of the heart: Apical (Apex), mid-ventricular (Mid), and basal (Base). Uncorrected and automatically corrected segmentations were compared to the expert corrected segmentations using two measures of spatial correspondence: Dice similarity coefficient (left) and Hausdorff distance (right).

More powerful transformation models for the registration method, such as spline or more general non-rigid registration, were not considered for this task since the aim of the proposed method was to correct for breath-hold motion misalignments 

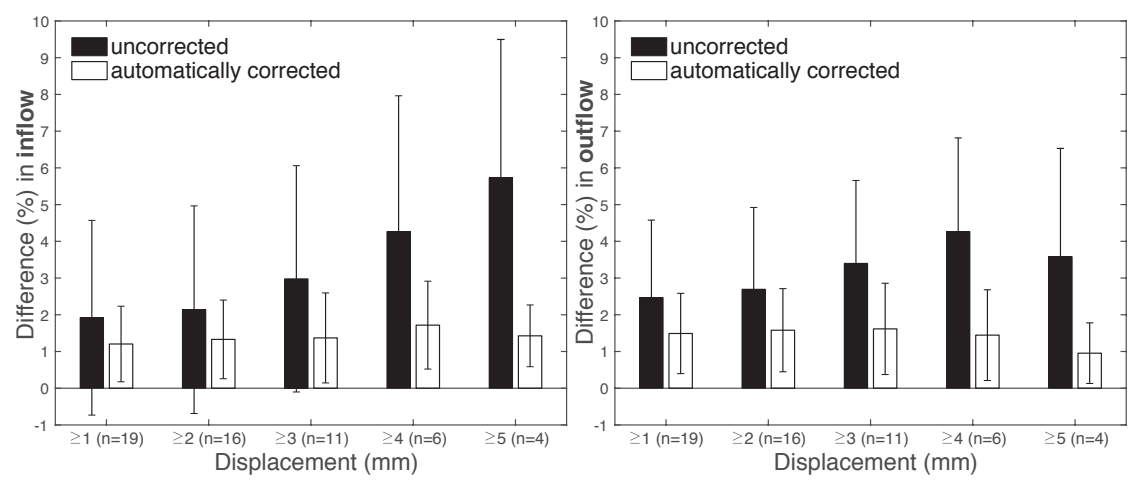

Figure 11: Comparison of flow volumes calculated using pathlines between the uncorrected (black) and the automatically corrected (white) segmentations. Inflow differences (left), outflow differences (right).

that typically present themselves as small rigid differences between short-axis slices. Additionally, non-rigid transformations might result in unwanted alterations in the anatomy depicted in the images. Large differences between the b-SSFP and the 4D Flow MRI caused by other types of motion or by acquisition issues were not the focus of this work and would most likely require different correction procedures.

The method does not require any manual input, it is fairly quick, and can be used to correct data that has already been segmented; therefore, no extra segmentations or new image acquisitions are required. Consequently, the implemented correction method can be easily added to the pre-processing steps of 4D Flow MRI analysis.

\subsection{Vessel Segmentation}

Clinical blood flow based assessment with MRI is typically done by measuring blood flow passing through a 2D PC-MRI plane placed perpendicular to a vessel, manually or semi-automatically segmenting the vessel in each timeframe of the cardiac cycle, and calculating the flow volume over all these timeframes in order to assess the amount of blood that goes through the blood vessel during a heartbeat. Assessment of flow volumes in the great thoracic vessels can also be done using 4D Flow MRI, with the advantage of having the possibility to include and relocate as many analysis planes as necessary, without requiring any additional MR acquisitions [139-141]. However, as discussed in section 6.1, most vessel segmentation techniques intended for 4D Flow MRI have aimed for three-dimensional results and require a varying degree of manual inputs in order to generate adequate results.

In Paper II, “Atlas-based analysis of 4D flow CMR: Automated vessel segmentation and flow quantification", we propose the use of atlas-based segmentation with 4D Flow MRI to automatically segment the great thoracic vessels at each timeframe of the cardiac cycle, locate planes at predefined vessel positions, and calculate their 
corresponding flow volumes. The main goal of the study was to automate and simplify the post-processing steps required to generate a four-dimensional segmentation of the vessels, together with a general evaluation of the cardiac status of the subject done through vessel flow analysis.

For this study, an atlas was created by semi-automatically segmenting the aorta, pulmonary artery, and caval veins in one 4D Flow MRI dataset. Additionally, the atlas included plane locations at which flow volume calculations would be valuable for cardiac function evaluation. The image used to segment the vessels was an angiography of the atlas dataset created by including only the systolic timeframes of the 4D Flow MRI. The atlas can be seen in Figure 12, while Figure 13 shows the plane locations chosen for each vessel.

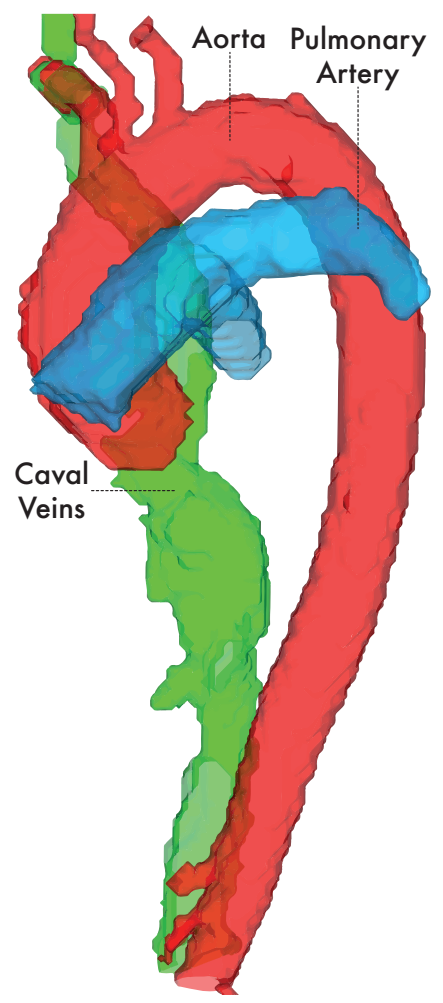

Figure 12: Atlas of the great thoracic vessels. Red: aorta, blue: pulmonary artery, green: caval veins. 


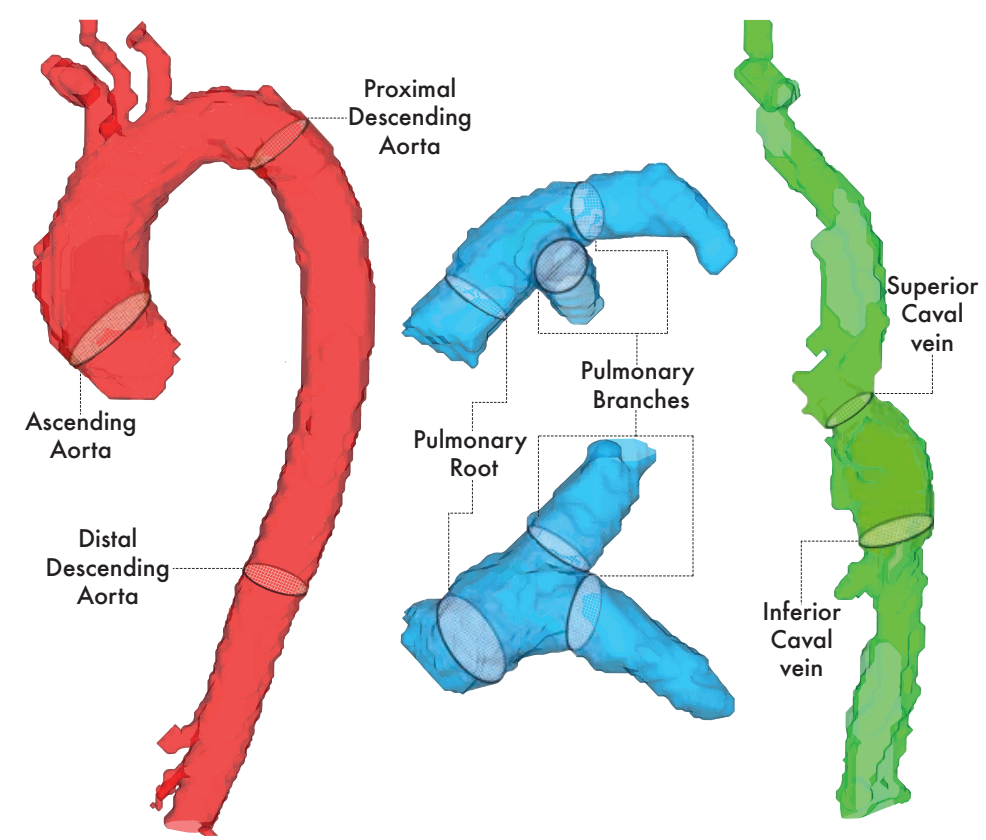

Figure 13: Great thoracic vessels included in the atlas visualized independently with their corresponding planes for flow volume analysis.

The automatic procedure to segment and assess a target 4D Flow MRI dataset can be described as follows:

1. As done previously for the atlas, an angiography of the systolic timeframes was generated using the input 4D Flow MR dataset.

2. The angiographies were registered and the resulting displacement field was applied to the atlas' segmentation labels to generate a segmentation for the target dataset. This segmentation includes labels for the major vessels at systole, and locations of the flow analysis planes.

3. A systolic timeframe, $t_{s}$, of the input dataset was chosen as the one corresponding to the segmentation generated in the previous step. The target's magnitude image at $t_{s}, M\left(t_{s}\right)$, was non-rigidly registered to all the available timeframes of $M$. The resulting deformations were applied to the segmentation generated in step 2. The final result was a four-dimensional segmentation of the vessels, also including the locations of the assessment planes.

4. Net flow volumes and volumetric flow rates at each timeframe were calculated for all the planes included in the segmentation.

Figure 14 shows the resulting segmentation generated for one subject, together with flow velocity visualization for all the vessels using instantaneous streamlines. 


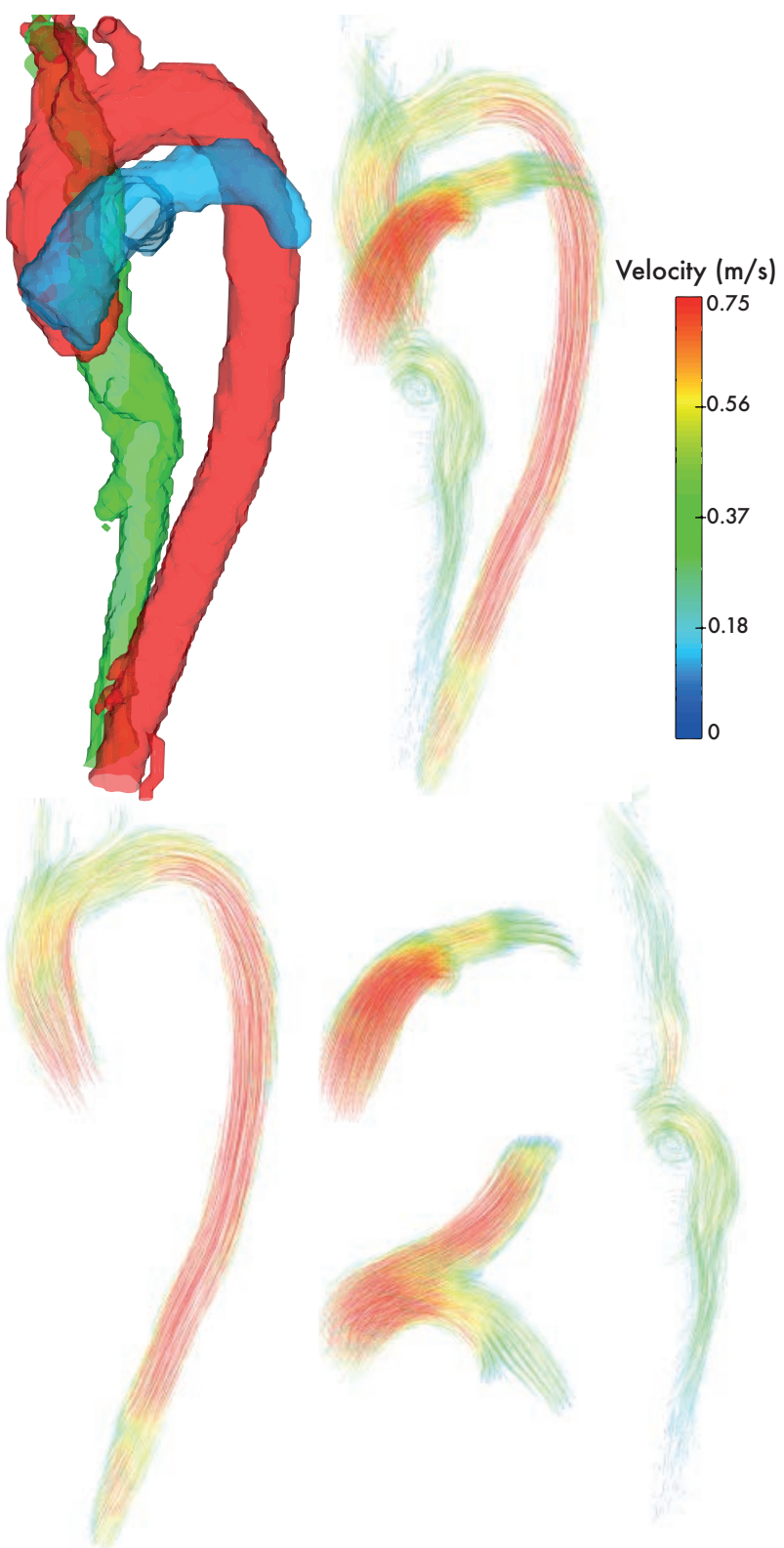

Figure 14: Segmentation generated with the proposed method at one systolic timeframe. Top: All the vessels visualized using isosurfaces (left), and instantaneous streamlines of the flow velocities (right). Bottom: Flow velocities for each vessel, aorta (left), pulmonary artery (center), and caval veins (right). 
Note that only one timeframe is shown in the figure due to space considerations; however, the segmentation generated is four-dimensional and includes all the timeframes available in the 4D Flow MR dataset.

To evaluate the method, 4D Flow MRI acquisitions were obtained from healthy volunteers and patients with mild or moderate heart failure for a total of 21 datasets. The results obtained in the ascending aorta were compared to manual segmentation of the same vessel performed in 2D PC-MRI planes, obtaining excellent correlation. Also, the flow volumes obtained at different locations were compared in order to ensure that the values obtained were coherent with the expected cardiovascular inputs and outputs. Good agreements were found between the flow volumes obtained in the ascending aorta, pulmonary trunk, pulmonary branches, and caval veins. Linear regresion analysis of these comparisons are shown in Figure 15.
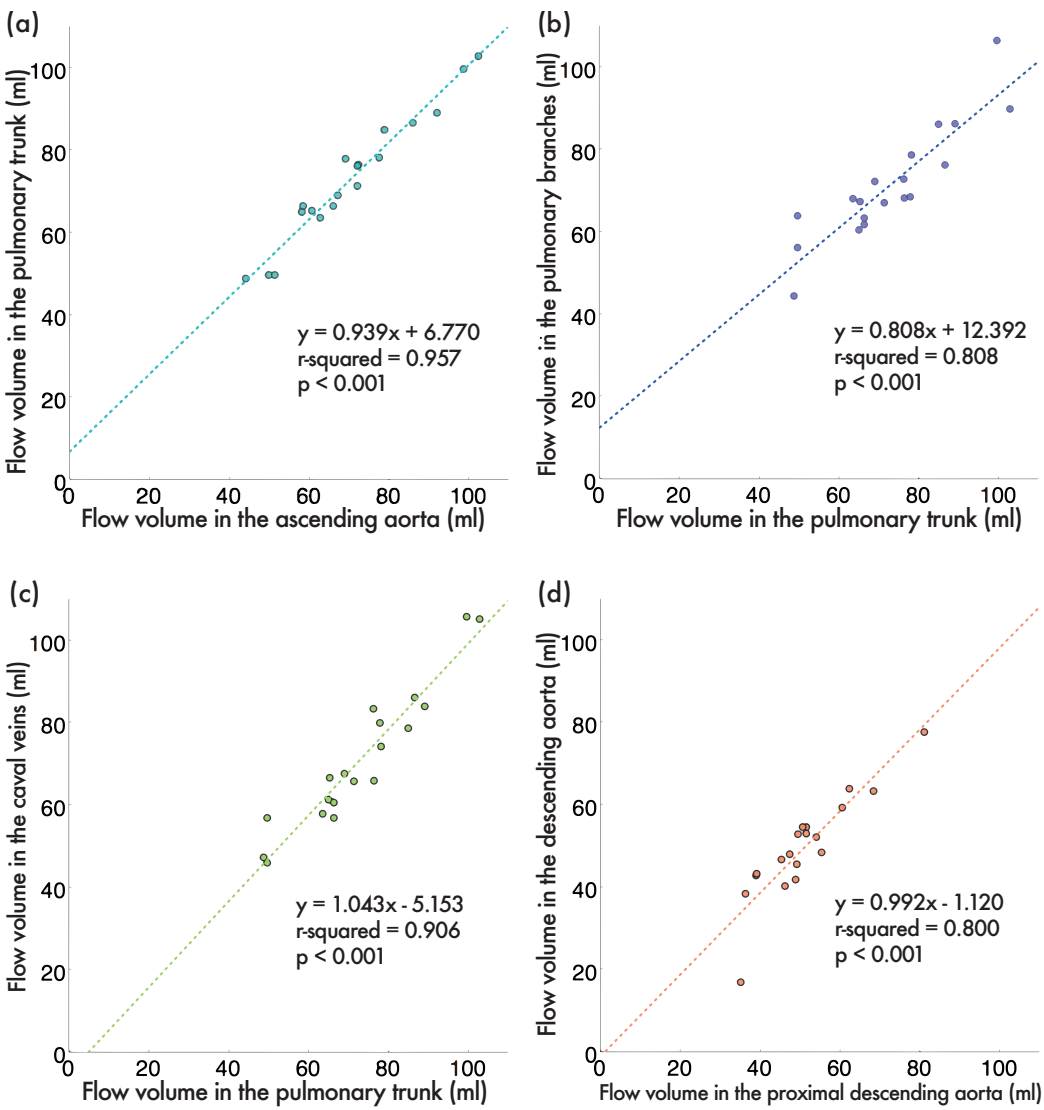

Figure 15: Linear regression analysis of the flow volumes calculated with the proposed method. (a) Ascending aorta vs. pulmonary trunk. (b) Pulmonary trunk vs. pulmonary branches. (c) Pulmonary trunk vs. caval veins. (d) Proximal descending aorta vs. distal descending aorta. 
Once an atlas has been created, the method is completely automatic and can be used as part of the post-processing and visualization techniques applied on the 4D Flow MRI datasets. Additionally, the four-dimensional segmentations generated could be used to visualize the motion of the vessels throughout the cardiac cycle, and could aid in the calculation of hemodynamic parameters that depend on the location of the vessel wall, such as wall shear stress.

The main limitation of the proposed method is the use of a single atlas to segment the vessels, whose morphology can potentially vary between distinct subjects. A new atlas, or set of atlases, would be required to segment and assess specific pathological vascular anatomies. We improved upon this method in Paper $\mathbf{V}$ by including multiple atlases in the segmentation process in order to account for more variability between subjects, in addition to generating a segmentation for the cardiac chambers. See section 7.5 for further details.

\subsection{Angiographic Visualization}

As discussed in section 4.5.3, Phase-contrast Magnetic Resonance Angiography (PCMRA) data can be generated by combining the blood flow velocity information and the magnitude data included in a 4D Flow MRI acquisition. However, the current standard for 4D Flow MRI angiographic data generation typically averages all the available timeframes into one three-dimensional image in which moving structures are difficult to perceive. This can impair visualization of the heart chambers, as well as the computation of parameters that rely on a precise segmentation, such as wall shear stress in the large arteries.

In Paper III, "Improving visualization of $4 D$ Flow CMR with four-dimensional angiographic data: Generation of a $4 D$ pase-contrast magnetic resonance CardioAngiography (4D PC-MRCA)", we propose an improvement over the method used for PC-MRA data generation from 4D Flow MRI. We present a technique for the generation of a temporally resolved Phase-Contrast Magnetic Resonance CardioAngiography (4D PC-MRCA) that includes the geometry of the lumen of the vessels as well as the heart over the entire cardiac cycle.

The procedure to generate a 4D PC-MRCA from a 4D Flow MRI dataset can be described as:

1. Generate a phase-contrast angiography without averaging the image data, thereby retaining the four-dimensional information included in the 4D Flow MRI dataset. We used equation (41) in order to take advantage of the particular settings of our datasets; however, this equation can be adapted accordingly, as suggested in previous studies [45].

$$
\operatorname{PC}-\operatorname{MRA}(t)=M(t) *\left(V_{x}^{2}(t)+V_{y}^{2}(t)+V_{z}^{2}(t)\right)^{\gamma}
$$

where $t$ represents each timeframe of the dataset, $M$ is the magnitude volume of the 4D Flow MR image, $V_{x}, V_{y}$ and $V_{z}$ are the velocity volumes in each of the three space directions, and $\gamma$ is a correction value experimentally set to 
0.2 to enhance the image intensities in areas of lower blood flow, such as the cardiac chambers.

2. The image generated in the previous step has considerable differences between the intensities of its different timeframes, a consequence of the cardiovascular system's pulsatile blood flow. In this step, we use non-rigid registration to align all the timeframes of the 4D Flow MRI magnitude volume to one chosen timeframe $\hat{t}$ of $M$. The result is a set of $N-1$ transformations, $B=\left\{B_{1}, B_{2}, \ldots, B_{N-1}\right\}$, for $N$ timeframes in the 4 D Flow MR dataset.

3. Each timeframe $t$ of the PC-MRA created in step 1 is transformed using its corresponding transformation $B_{t}$, resulting in a four-dimensional angiography in which all timeframes are morphologically located at timeframe $\hat{t}$, but with with different blood flow intensities depending on their position in the cardiac cycle.

4. Generate a maximum intensity projection (MIP) of the four-dimensional image generated in the previous step, resulting in a three-dimensional PC-MRCA. This step is comparable to the averaging step included in the previously published PC-MRA generation techniques; however, in this case we are able to identify the particular timeframe to which the PC-MRCA belongs to at this point, i.e., the chosen timeframe $\hat{t}$.

5. Calculate a new set of transformations $F=\left\{F_{1}, F_{2}, \ldots, F_{N-1}\right\}$ using non-rigid registration between $M(\hat{t})$ and the remaining timeframes of $M$. As in step 3 , the transformations are applied to the 3D PC-MRCA resulting in a fourdimensional cardioangiography.

Figure 16 shows a 4D PC-MRCA at one mid-diastolic timeframe using different visualization methods. The 4D PC-MRCA at different timeframes of the cardiac cycle is depicted in Figure 17.

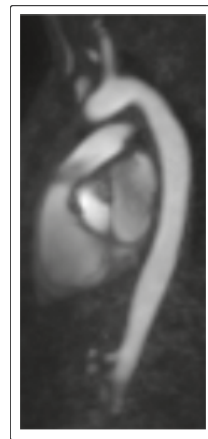

(a)

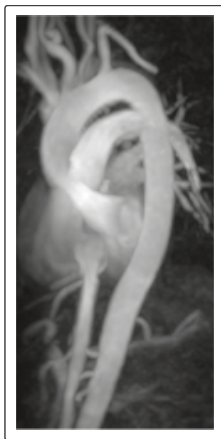

(b)
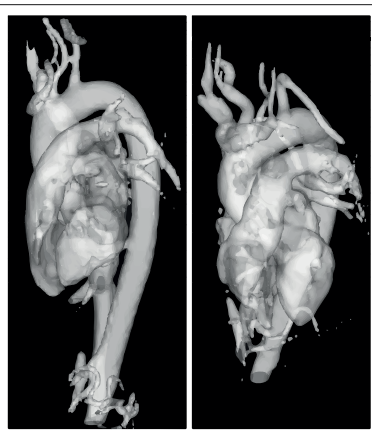

(c)

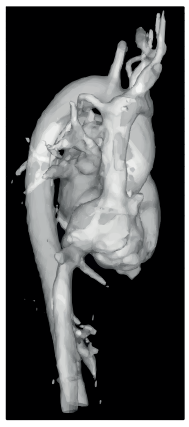

Figure 16: 4D PC-MRCA at mid-diastole visualized as: (a) a coronal slice, (b) a maximum intensity projection, (c) an isosurface from different points of view. 


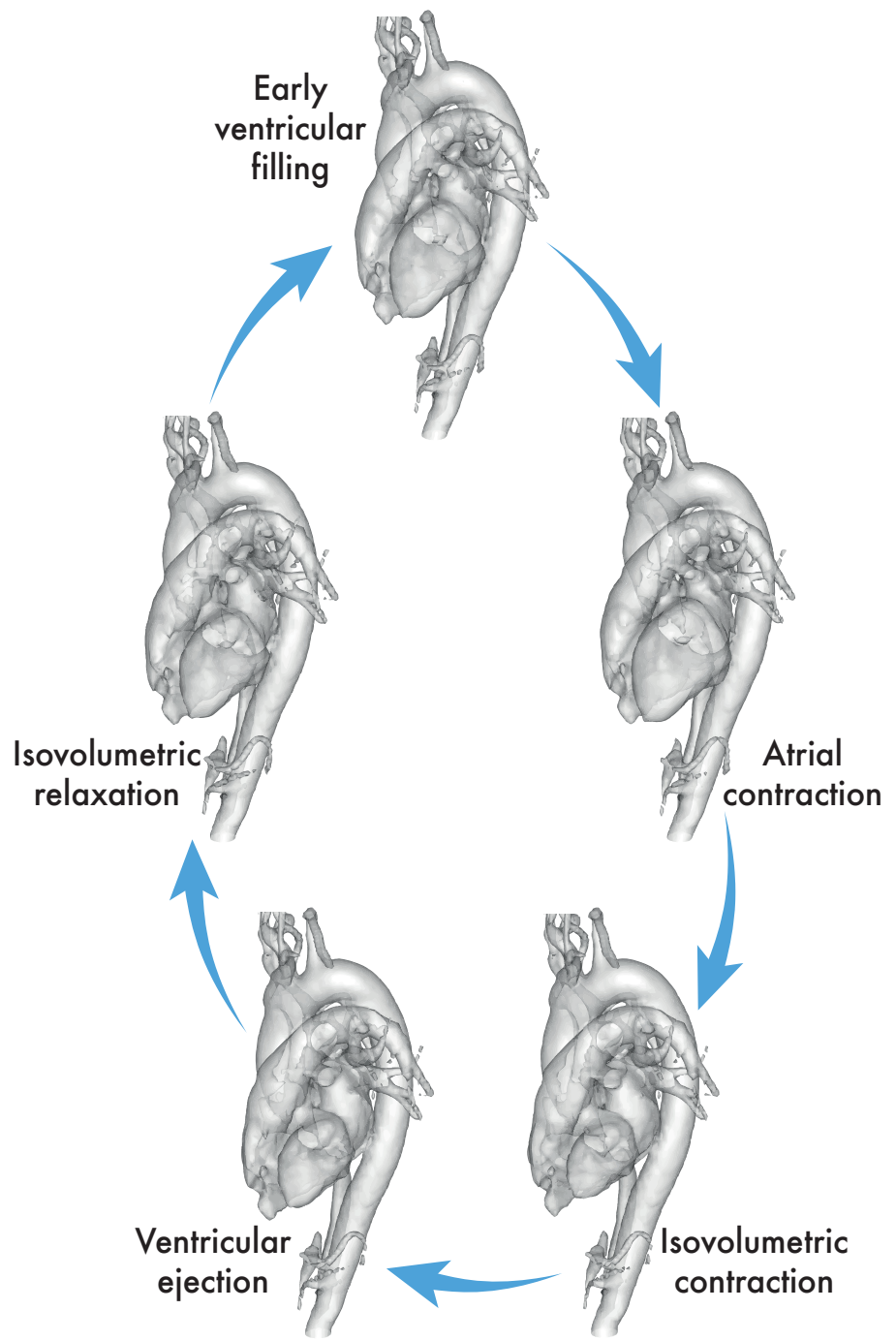

Figure 17: Several timeframes of the cardiac cycle visualized using isosurface renderings of the 4D PC-MRCA

The method was visually evaluated in a group of ten healthy volunteers with no prior history of cardiovascular disease. The 4D PC-MRCAs were compared to 3D PC-MRA data generated using equation (42), previously described in [46].

$$
\operatorname{PC-MRA}(t)=\sqrt{\frac{1}{N} \sum_{t=1}^{N} M^{2}(t) *\left(V_{x}^{2}(t)+V_{y}^{2}(t)+V_{z}^{2}(t)\right)}
$$


Two experts scored maximum intensity projections of the 4D PC-MRCAs and 3D PC-MRAs based on visibility of anatomical regions for clinical assessment. For this purpose, we devised an image quality scale from 1 to 4 based on criteria that we considered would be most important for cardiovascular diagnosis. The resulting scores were higher for the PC-MRCAs in all the regions evaluated. Additionally, noticeable discrepancies with respect to the cardiovascular morphology were observed on the PC-MRAs on a considerable number of timeframes throughout the cardiac cycle due to cardiac and vessel motion.

In addition to offering an improved visualization of the heart and vessels over the cardiac cycle, the 4D PC-MRCAs can also be used in order to compare or register different 4D Flow MRI datasets to each other. This feature was used in Paper IV to create an average dataset from a group of subjects, and in Paper $\mathbf{V}$ to generate automatic four-dimensional segmentations of the cardiac chambers and vessels (see sections 7.4 and 7.5).

\subsection{Assessment of Hemodynamic Parameters}

A current challenge in the assessment of 4D Flow MRI data is the lack of automatic methods that are able to handle the extensive amount of information available when considering a large group of datasets, or when comparing sizable groups of datasets to each other, each with particular characteristics. Moreover, the desired methods should also be able to extract complex knowledge from these datasets without reducing the amount of information to a single value, as it is quite common when analyzing 4D Flow MR images.

In Paper IV, "Creating Hemodynamic Atlases of Cardiac 4D Flow MRI”, we developed a framework for cohort-based blood flow assessment of 4D Flow MRI data, with the main objective of improving the feasibility of cohort studies based on this type of MR acquisition. A cohort-averaged hemodynamic atlas was created from a set of healthy volunteers in order to visualize and assess the group-wise behavior of hemodynamic parameters during the cardiac cycle.

The technique developed in Paper III was used to generate 4D PC-MRCAs for all datasets, and these images were used to generate the cohort's averages. Creating average images or volumes for a group of datasets has been done previously with different objectives in mind, such as, hemodynamic analysis, model-based segmentation, and statistical shape analysis $[13,64,142]$. In this study, the procedure to create an average volume for a cohort was the following:

1. PC-MRCAs were generated for each of the datasets in the cohort. See section 7.3 for details on how to generate a PC-MRCA from a 4D Flow MRI. These images were used to register the datasets to each other.

2. The PC-MRCAs were temporally aligned to each other with respect to each subject's cardiac cycle. To do this, the velocities included in the 4D Flow MRI were used to define three specific phases in the cardiac cycle: mid-systole, 
early-diastole, and end-diastole. Subsequently, these timeframes were used to temporally align the 4D PC-MRCAs, using interpolation to generate the intermediate frames so that all the datasets consisted of 40 timeframes.

3. One subject, referred to as "Subject 1" $\left(S_{1}\right)$, was randomly selected to be the base of the average volume. The PC-MRCAs of all datasets were rigidly registered to $S_{1}$ to reduce possible rotation or translation differences between the images.

4. Using non-rigid registration, the PC-MRCA of $S_{1}$ was registered to the PCMRCAs of all the datasets in the cohort obtained in the previous step.

5. The deformation fields obtained in step 4 were averaged into one four dimensional displacement field that was applied to $S_{1}$, resulting in an average volume, or "average heart". Since the images were temporally aligned in step 2 , all the registrations were done for each timeframe available. Consequently, the generated average volume is also four-dimensional.

Once created, the average volume was used to generate hemodynamic atlases of specific parameters as follows:

1. A hemodynamic parameter to be assessed was selected and calculated for each of the 4D Flow MR datasets in the group. The output of this step is a four-dimensional image for each dataset in which each voxel's value is the chosen hemodynamic parameter calculated for that voxel.

2. All the PC-MRCAs of the group were non-rigidly registered to the average. The resulting displacement fields were applied to the hemodynamic volumes generated in the previous step.

3. The registered hemodynamic volumes were averaged to obtain the hemodynamic atlas for the chosen parameter. Atlas volumes were also created using the standard deviation of the hemodynamic parameter in order to visualize the areas where group values differ.

4. Regions of interest were segmented in the average volume, using the resulting segmentation on the hemodynamic atlas. For this study, we segmented the left ventricle, left atrium, and ascending aorta at three timeframes: mid-systole, early-diastole, and end-diastole.

The proposed method was evaluated in a group of thirteen 4D Flow MR datasets acquired from healthy volunteers with no current or prior history of cardiovascular disease. Hemodynamic atlases of kinetic energy $(\mathrm{KE})$ and helicity density $\left(H_{d}\right)$ were generated for the group, and the effect of registration on these parameters was evaluated. Volume renderings of the hemodynamic atlases for $\mathrm{KE}$ and $H_{d}$ at three timeframes of the cardiac cycle can be seen in Figure 18.

Registration was applied to each dataset individually to evaluate the effect of displacement and interpolation on the hemodynamic parameters. We found that the 


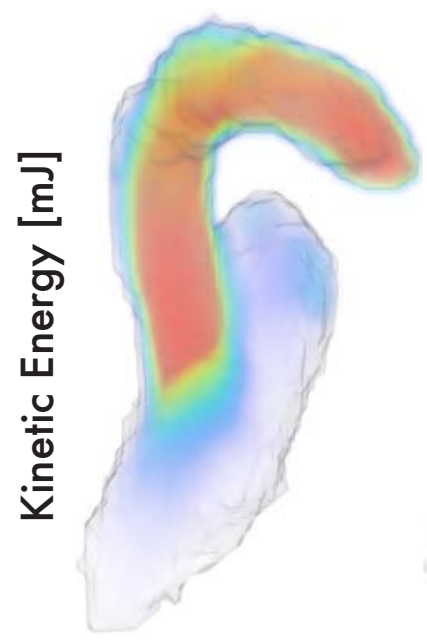

Mid-systole

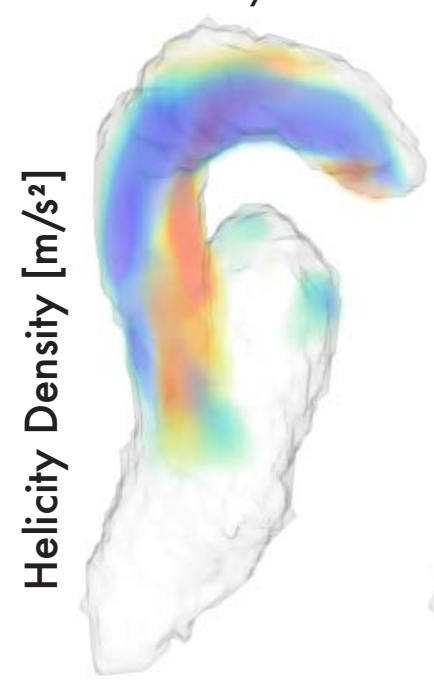

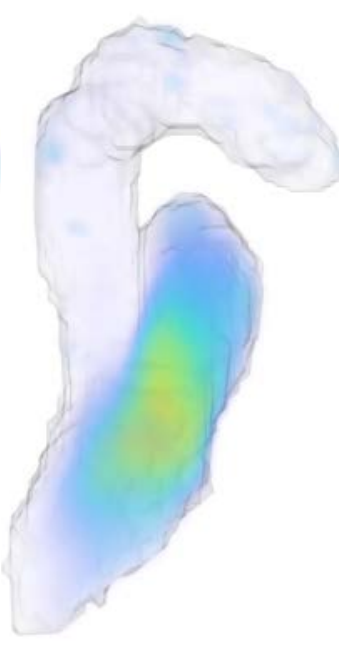

Early diastole

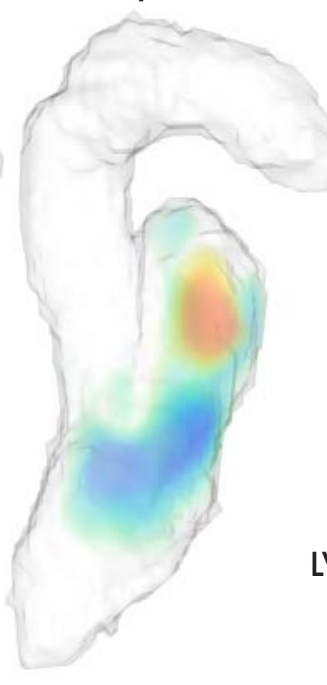

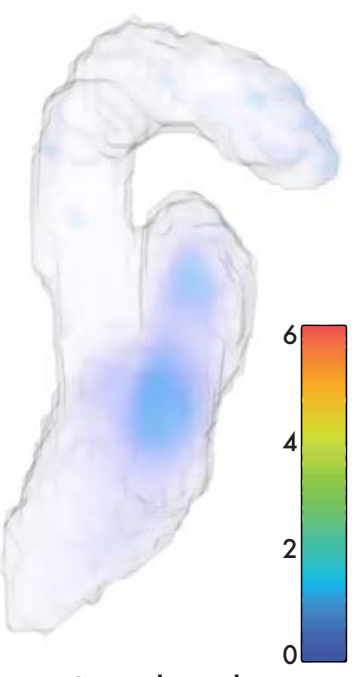

Late diastole

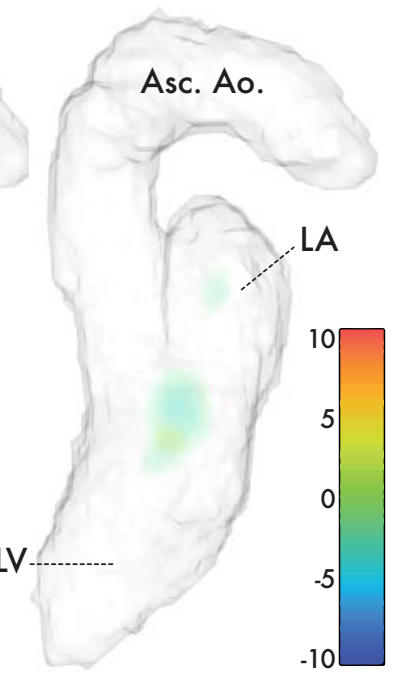

Figure 18: The kinetic energy and helicity density atlases at three timeframes of the cardiac cycle including the left ventricle (LV), left atrium (LA) and the ascending aorta.

peak values were slightly underestimated after registration. However, the general value distribution of the parameters remained the same after they were registered, and the mean values were also not significantly affected.

The accuracy of the average volume generation method was assessed by creating additional manual segmentations of the cohort's PC-MRCAs, using non-rigid, rigid, and affine registration between these PC-MRCAs and the average volume, and 
comparing the overlap of these new registered segmentations with the segmentation of the average volume. Additionally, rigid and affine registration methods were evaluated for step 2 of the hemodynamic atlas creation in order to determine the benefit of using non-rigid registration in this step. Non-rigid registration was found to generate better results in all cases.

The proposed method is mostly automatic, requiring only the segmentation of the group's average volume. Segmentation of each individual dataset is not necessary, thereby avoiding a considerable amount of time-consuming work.

The approach developed in this paper could be used to generate disease-specific hemodynamic atlases of patient groups with different cardiovascular disorders. The technique could also be applied to assess other valuable parameters such as turbulent kinetic energy, energy loss, vorticity, flow speed, among others, in any region of interest included in the 4D Flow MR image.

\subsection{Cardiac Segmentation}

Segmentation of the cardiac chambers and great thoracic vessels is one of the most important and simultaneously difficult challenges to resolve when assessing 4D Flow MR images. As discussed in section 6.2, the current standard for cardiac 4D Flow MR segmentation makes use of b-SSFP MR images of the heart by delineating the regions of interest in these images. However, the use of cardiac b-SSFP MR for this purpose affects the resulting segmentations negatively due to the inherent characteristics of this acquisition. On the other hand, direct manual or semi-automatic segmentation on 4D Flow MR images has frequently been deemed unfeasible due to a number of reasons:

- Their relatively lower resolution when compared to other cardiac MR acquisitions. This typically results in partial volume effects which can make structures like the myocardium, papillary muscles, or cardiac valves difficult to distinguish.

- The sheer amount of data included in the images. A comprehensive segmentation requires a three-dimensional segmentation per timeframe for each region of interest, which ideally should include the cardiac ventricles and great thoracic vessels.

In Paper V, “Automated Multi-atlas Segmentation of Cardiac 4D Flow MRI”, we propose and evaluate an automatic method to segment the cardiac chambers and great thoracic vessels in 4D Flow MRI data. The technique is based on multiatlas segmentation, and, similarly to Paper IV, takes advantage of the information available on the 4D PC-MRCAs presented in Paper III in order to register different 4D Flow MR datasets to each other.

A group of atlases was created by segmenting eight 4D Flow MR datasets using semi-automatic methods. Each atlas included labels for the four cardiac chambers, aorta, and pulmonary artery at two timeframes: end-systole $\left(t_{E S}\right)$ and end-diastole $\left(t_{E D}\right)$. Figure 19 shows two of the atlases used in the study. 


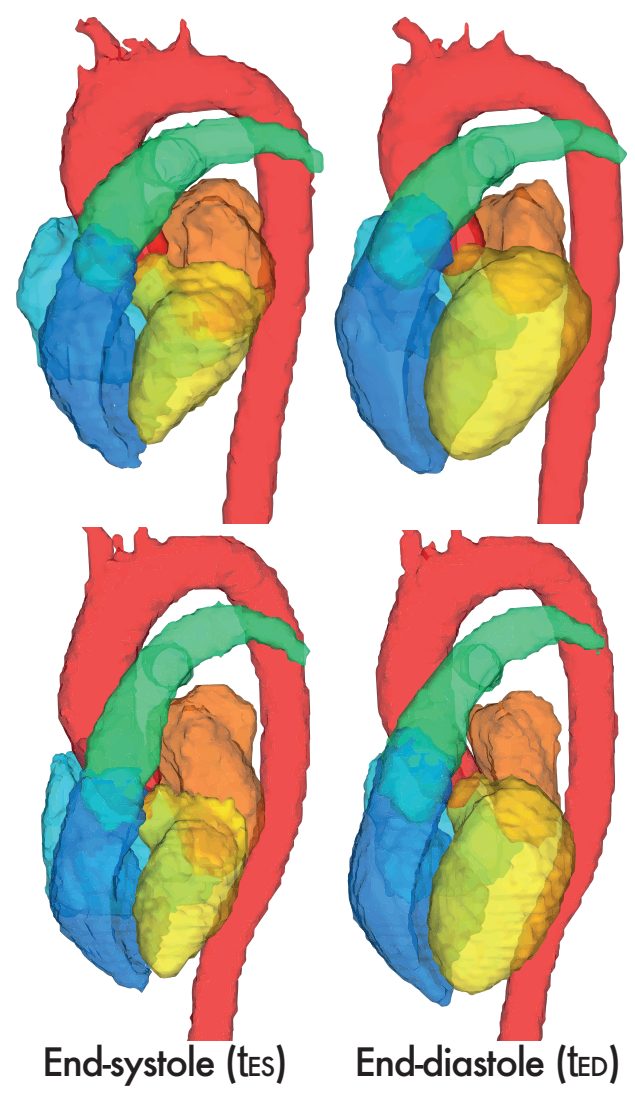

Figure 19: Labels created using semi-automatic methods on two atlases at end-systole and end-diastole. Red: aorta, yellow: LV, orange: LA, green: pulmonary artery, blue: RV, cyan: RA.

A 4D PC-MRCA was generated for each atlas. Subsequently, to segment a target 4D Flow MRI, the method was a follows:

1. Generate a 4D PC-MRCA for the target dataset using the technique described in section 7.3.

2. Apply multi-atlas segmentation independently for $t_{E S}$ and $t_{E D}$ with the following steps:

(a) Register the 4D PC-MRCA of each atlas to the 4D PC-MRCA of the target dataset at $t_{E S}$ and $t_{E D}$. For this step, affine registration was applied first, followed by non-rigid registration.

(b) Apply the displacement fields corresponding to each atlas to its labels, resulting in a set of possible segmentations for the target dataset at the 
chosen timeframes.

(c) Fuse the eight available solutions into one segmentation for each of the selected timeframes, $t_{E S}$ and $t_{E D}$. To this end, we used the STAPLE algorithm, described in section 5.2.1, to calculate a probabilistic estimate of the true segmentation for each timeframe.

3. Propagate each of the generated segmentations in time as follows:

(a) Register the target's magnitude at $t_{E S}, M\left(t_{E S}\right)$, to all timeframes to generate a set of displacements fields, one for each of the $N$ available timeframes. Similarly for $M\left(t_{E D}\right)$. Note that these displacement fields were already calculated during step 1 .

(b) The displacement fields were applied to the segmentations obtained in step 2(c) to create two possible segmentations, each based on one of the chosen timeframes, $t_{E S}$ and $t_{E D}$.

4. Create a final segmentation by combining these two segmentations into one final result. The segmentation created from $t_{E S}$ was preferred during systolic timeframes, and its influence was made to decrease as we got farther from endsystole. Correspondingly, the segmentation based on $t_{E D}$ had less influence during systole, and its importance increased gradually towards end-diastole.

Figures 20 and 21 show the segmentation results for three 4D Flow MRI datasets at end-systole, mid-diastole, and end-diastole visualized using isosurfaces from two different points of view.

The proposed method was evaluated on a group of 110 datasets acquired from both healthy volunteers and patients with suspected or diagnosed ischemic heart disease. End-systolic and end-diastolic volumes obtained automatically with the proposed technique versus those delineated manually on b-SSFP MR images were compared for the left ventricle and the left atrium. Figure 22(a-b) shows the results for the left ventricle. The correction method introduced in Paper I (Section 7.1) was used in order to minimize the effect of misalignments in the short-axis images on the manual segmentations. As an additional evaluation measure, flow volume analysis planes located in the ascending aorta and pulmonary artery root were generated using the technique described in Paper II (Section 7.2). These flow volumes were subsequently compared to the stroke volumes calculated using the segmentations of the left and right ventricles (see Figure 22(c-d)). Limits of agreement for all the values evaluated were lower or around $30 \mathrm{ml}$, which is similar to the results reported by previously published automatic segmentation techniques focusing on cardiac MRI [108, 143, 144].

The presented technique allows for fast and automatic segmentation of all the regions of interest included in a 4D Flow MRI dataset and can facilitate visualization and assessment of hemodynamic parameters in these images. Figures 23 and 24 use the segmentations shown in Figures 20 and 21 to visualize blood flow velocities throughout the cardiac cycle for three datasets. The flows are depicted using instantaneous streamlines at each timeframe. 

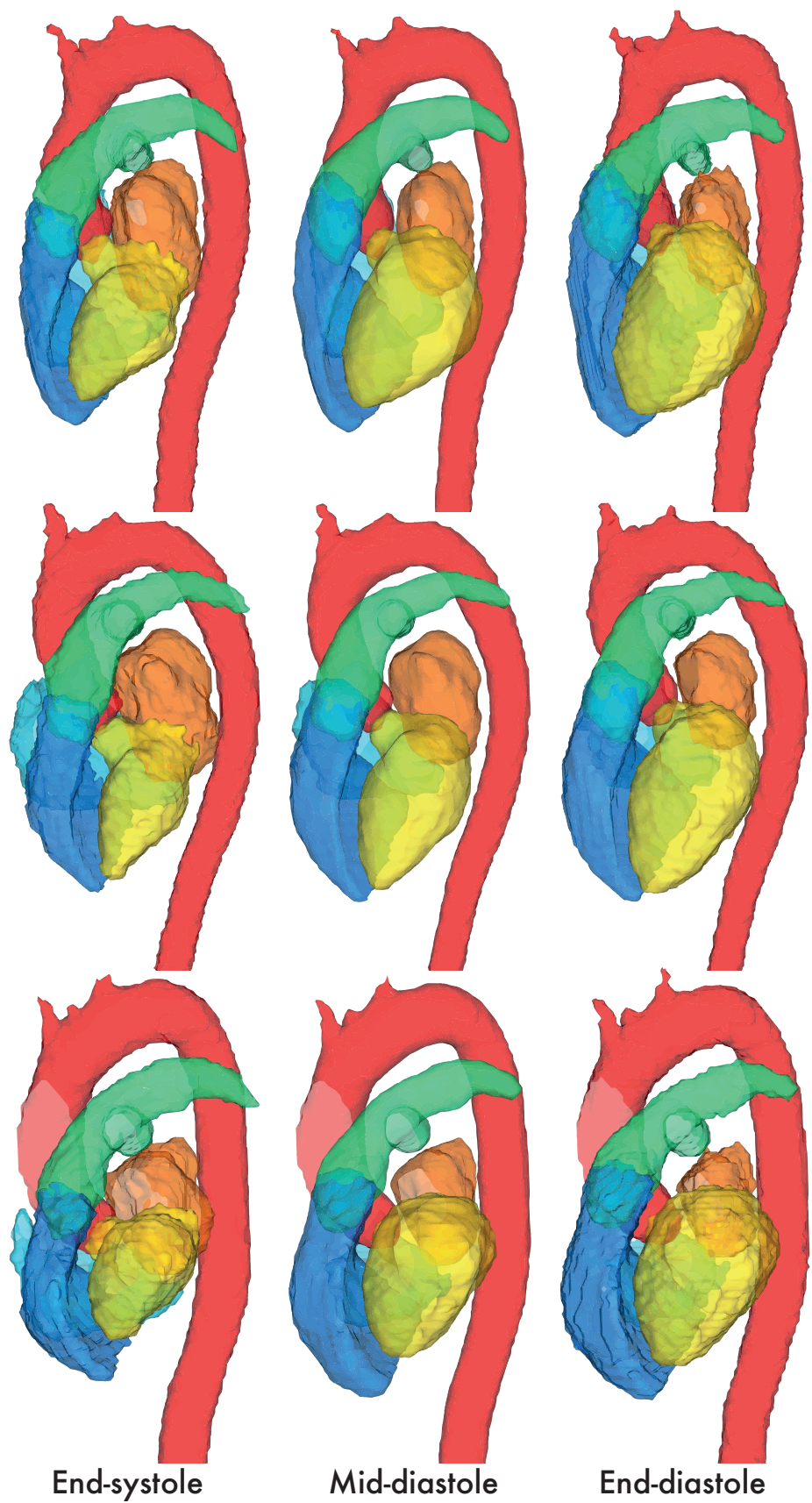

Mid-diastole

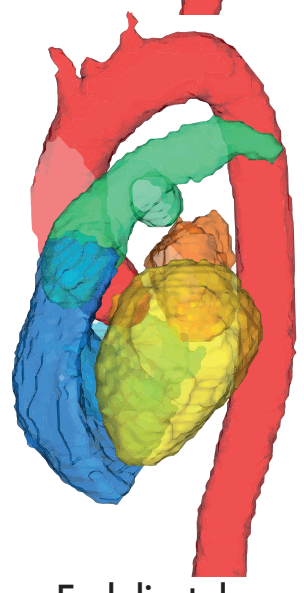

\section{End-diastole}

Figure 20: Segmentations generated for three datasets at three timeframes of the cardiac cycle visualized using isosurfaces. Red: aorta, yellow: LV, orange: LA, green: pulmonary artery, blue: RV, cyan: RA. 

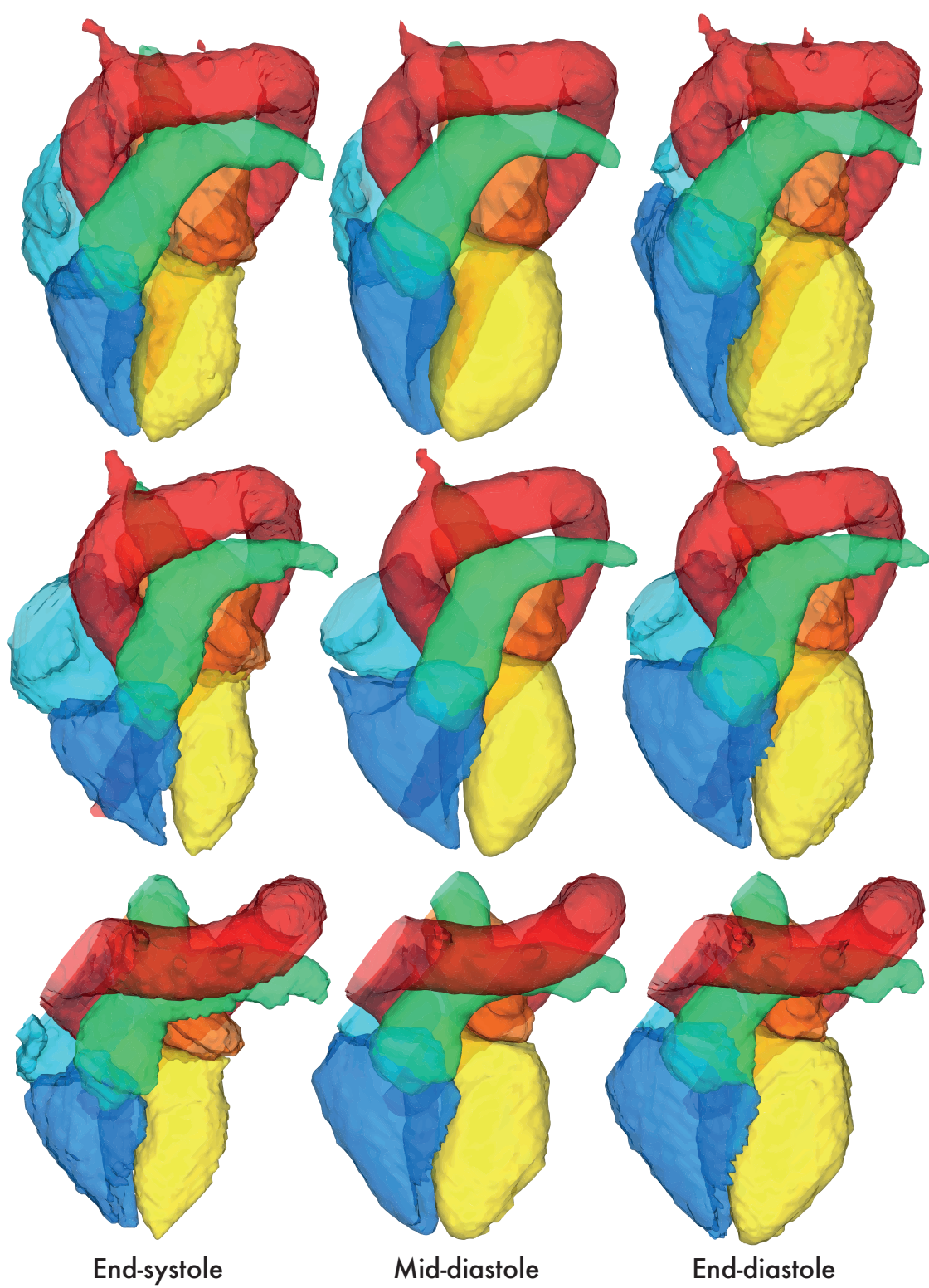

Figure 21: Segmentations generated for three datasets at three timeframes of the cardiac cycle visualized using isosurfaces. Red: aorta, yellow: LV, orange: LA, green: pulmonary artery, blue: RV, cyan: RA. 

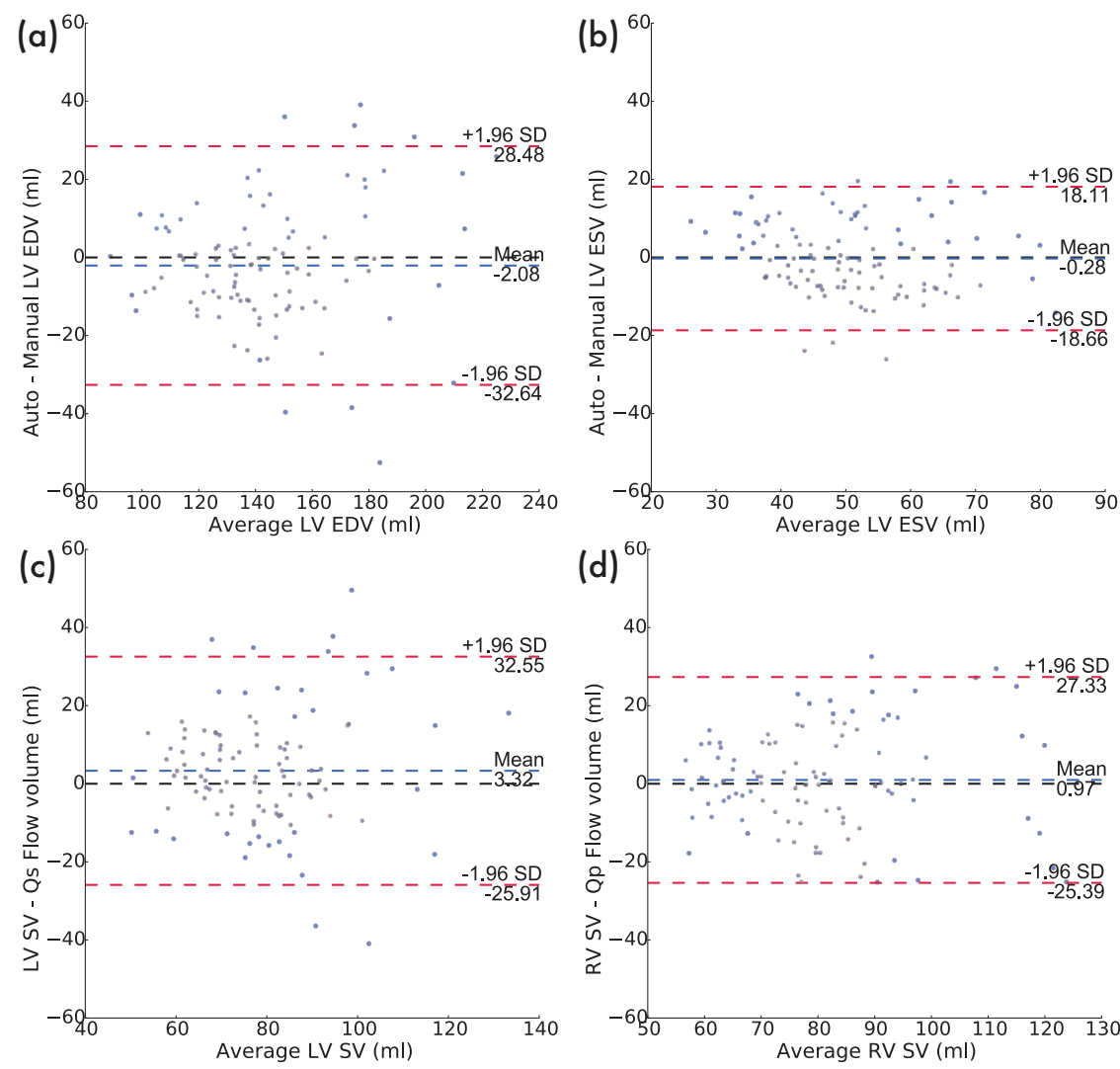

Figure 22: Blandt-Altman plots corresponding to the comparison of: (a) manual versus automatic segmentations of the LV EDV, (b) manual versus automatic segmentations of the LV ESV, (c) automatic LV SV versus systemic flow volume $\left(Q_{s}\right)$, and (d) automatic RV SV versus pulmonary flow volume $\left(Q_{p}\right)$ 


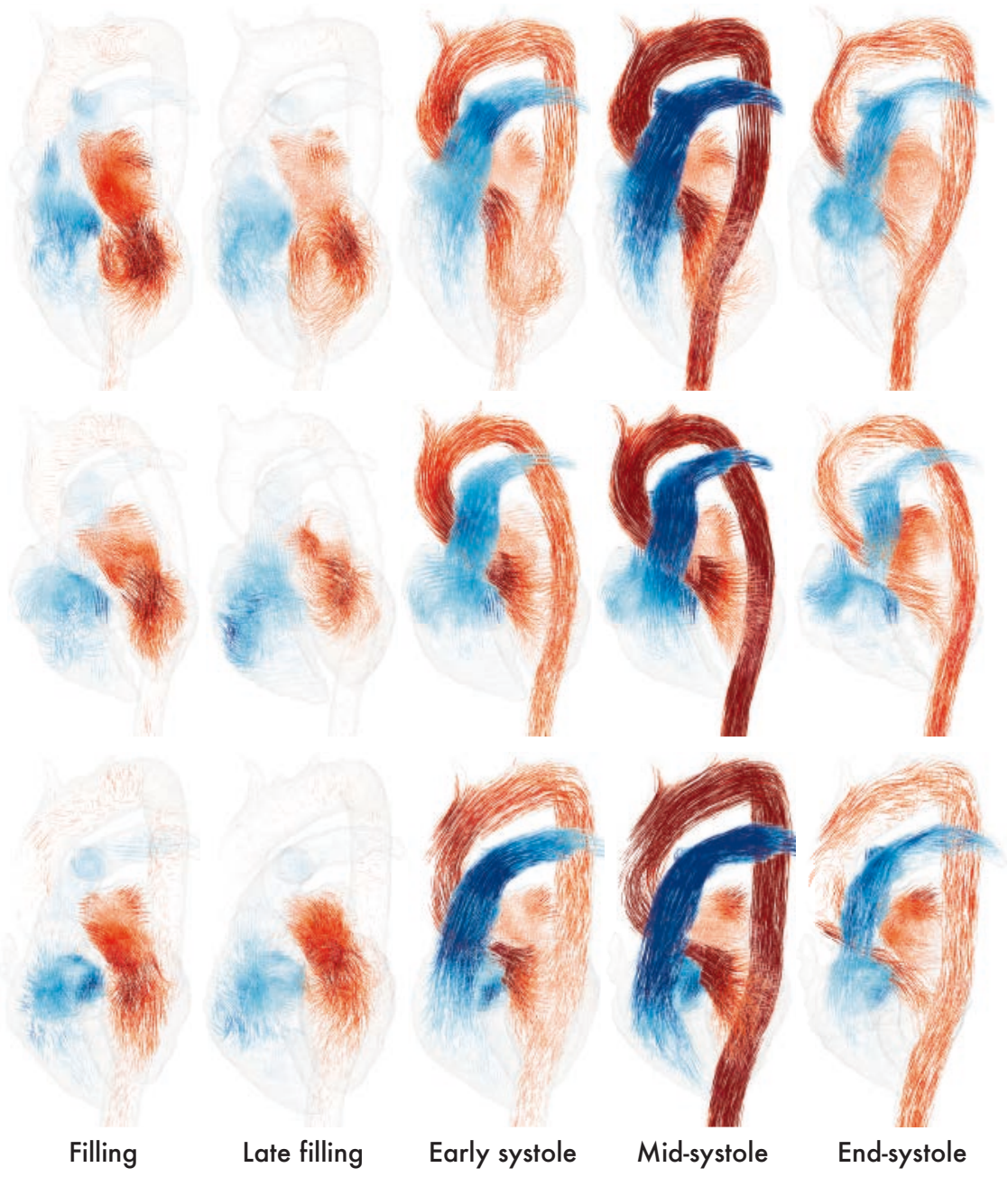

Figure 23: Flow visualization using the segmentations generated for three datasets at five phases of the cardiac cycle. The pulmonary flows are shown in shades of blue, and the systemic flows in shades of red. 

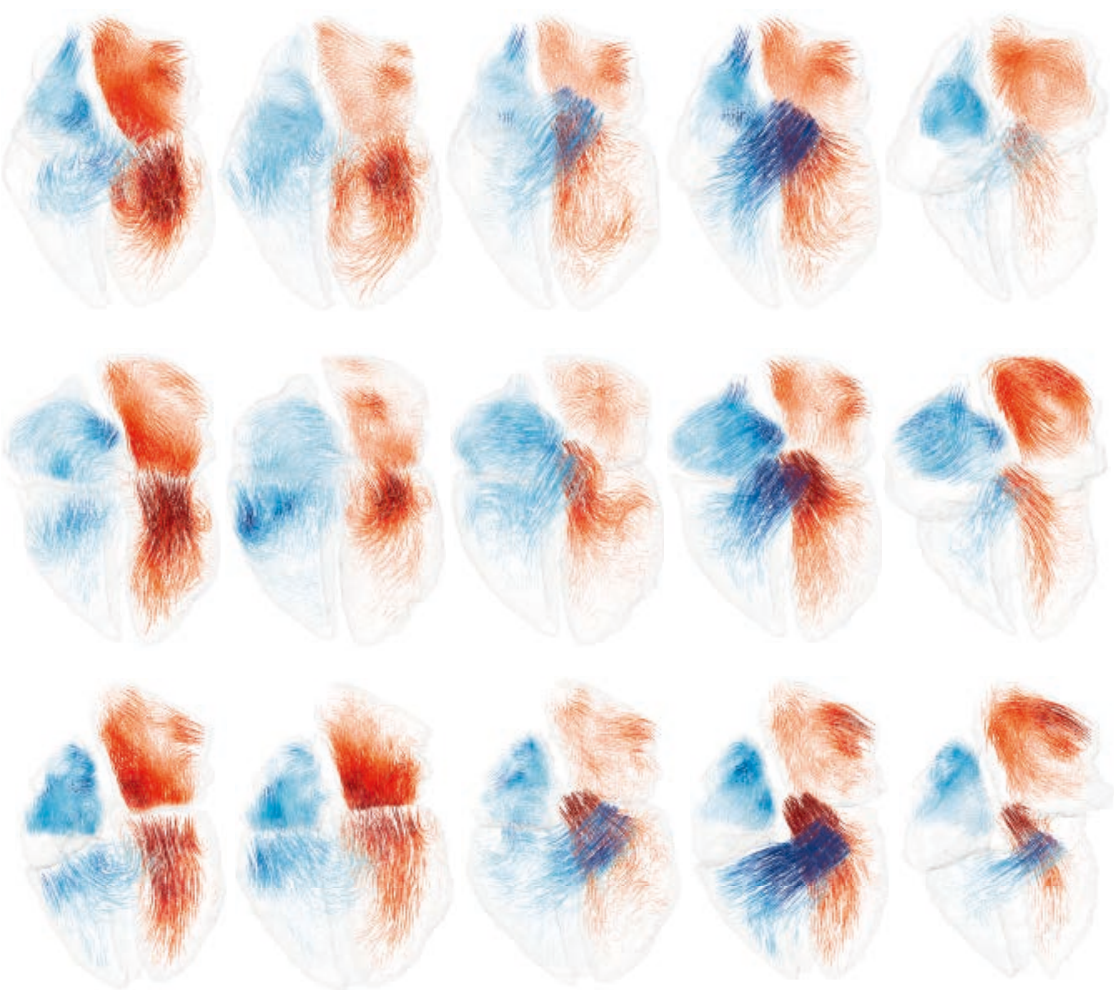

Filling

Late filling

Early systole

Mid-systole

End-systole

Figure 24: Cardiac flow visualization using the segmentations generated for three datasets at five phases of the cardiac cycle. The pulmonary flows are shown in shades of blue, and the systemic flows in shades of red. The heart is displayed using a four-chamber point of view. 



\section{Chapter 8}

\section{Summary and Future Outlook}

\subsection{Contributions of this thesis}

The main contribution of this thesis is the application and evaluation of novel image analysis techniques for the assessment 4D Flow MRI data, a complex and extensive category of MR acquisition. Accordingly, we have developed a series of tools to automate and assist an image analyst or physician throughout the process of visualizing and quantifying the valuable information included within these images. All the tools require minimal or no interaction from the user, with the aim of focusing the majority of the expert's analysis time in the assessment of the 4D Flow data, instead of during mandatory image preprocessing tasks.

Research done using 4D Flow MRI frequently focuses on regions with stronger blood flow dynamics, such as the cardiac left ventricle and aorta. Additional regions, such as the cardiac atria, right ventricle, and pulmonary artery are generally also included in the images, although they have typically been the focus of fewer studies. In Papers III, IV, and V, we presented techniques that can be used to visualize and assess any region of interest included in the 4D Flow MRI datasets.

In Papers IV and V we focused on cohort analysis from two points of view commonly used in medical research: analysis of groups of subjects with similar characteristics, and individual assessment per subject. These automatic tools for the analysis and segmentation of 4D Flow MR images facilitate the assessment of large cohorts of data, and can be valuable assets when aiming to obtain clinically significant results.

\subsection{Comments on the Current Implementation}

Image registration is a very popular subject within the research of medical imaging; consequently, thousands of methods and method variations have been proposed over the past decades. Section 5.1.1 contains a description of the registration techniques used during this work, which were chosen and evaluated for our specific aims. Nevertheless, the proposed methods are not constrained to these particular registration techniques in order to accomplish their goals, and any sufficiently adequate registration technique could likely be used for each case instead of the proposed 
ones.

During the development of most of the presented methods, we focused on establishing their feasibility when applied to 4D Flow MRI datasets. Consequently, although some efforts were made to use parallel programming when suitable, we did not focus on reducing the execution time of the algorithms as much as possible. The execution times required per-dataset for each of the proposed methods are the following:

- Paper I: $\sim 1$ minute

- Paper II: $\sim 10$ minutes ${ }^{1}$

- Paper III: $\sim 10$ minutes $^{2}$

- Paper IV: 20 hours. Parallel computing was used for the generation of 4D PC-MRCAs, but not during the calculation of the average atlas or the hemodynamic atlas

- Paper V: $\sim 25$ minutes

Further improvements could be done in this regard; for example, using GPU processing or cloud computing to help in the execution of the most demanding portions of the techniques, such as registration or hemodynamic parameter calculation.

Notably, most of the results are saved to disk after the first execution, and subsequent runs require only a few seconds. This is specially true for the techniques presented in Papers II and V, where the addition of new flow analysis planes or regions of interest to be segmented after the first run will be completed in a matter of seconds.

\subsection{Evaluation of Results}

A very important factor when developing image analysis tools is the evaluation of the generated results. This evaluation process depends almost entirely on the nature of the problem solved, but it typically incorporates a series of similarity measures to compare the results to a chosen ground truth.

In Paper III, we developed a technique for the generation of four-dimensional angiographic data from 4D Flow MRI. To evaluate this method, we considered a previous technique to generate angiographic images from 4D Flow MRI as ground truth for a visual quality assessment. This evaluation technique can be somewhat subjective, and could be influenced by external factors such as quality and brightness of the display used, degree of experience of the image expert, and relevance of the scoring technique. Nevertheless, we attempted to make the evaluation as trustworthy as possible by including two experts in the evaluation process and comparing their agreement, establishing an appropriate scoring scale, and providing a suitable environment for the evaluation. A perhaps more exhaustive evaluation could have

\footnotetext{
${ }^{1}$ Execution times reported in Paper II didn't use parallel programming.

${ }^{2}$ The implementation reported in Paper III was improved after publication to include more parallelization.
} 
been done by pixelwise assessment of the blood pool boundary at different locations throughout the image. However, the main goal of this study was to provide with an angiographic image that could be used for visualization of the blood pool, as opposed to a segmentation consisting of precise boundary locations.

Paper V, focusing on cardiac segmentation, used comparison to manual segmentations to evaluate the volumes in two ways: by comparing cardiac volumes at specific timeframes, and by calculating similarity between the volumes. Volume comparison can be a quite reliable evaluation measure, provided that the entire volume of interest is included in both MR acquisitions. Alternatively, using an overlap or distance measure between the ground truth and the generated segmentation is very commonly used in the medical imaging field to evaluate segmentation results. For this specific case, however, manual segmentation of 4D Flow MRI images on the scale necessary to sufficiently evaluate this method was deemed unfeasible. Consequently, for the left ventricle and atrium, the similarities were calculated using manual segmentations obtained using a different MR acquisition method-i. e., bSSFP MR. The main disadvantages of using b-SSFP for this purpose were described in Section 6.2. Additionally, similarity measures such as Dice Similarity Coefficient and Hausdorff Distance can also be impacted negatively, resulting in unfair outcomes for the evaluation.

In Papers I, II, and V, we took advantage of the flow velocities contained in the 4D Flow MR datasets and used this information as part of the method's assessment. This evaluation technique only depends on the 4D Flow MR image, and was done automatically in all cases using the technique presented in Paper II. Consequently, it was reliable, easy to reproduce, and unaffected by human error. Moreover, automatic flow volume analysis at different locations could also be used as a quality control measure for the 4D Flow MRI datasets to detect excessive noise, phase wrapping, or background correction errors.

\subsection{Use of Contrast Agents}

The 4D Flow MRI datasets used in Papers I, II, III, and V were obtained a short time after a CE-MRA acquisition that required the injection of a non-blood pool gadolinium-based contrast agent. Consequently, these 4D Flow MR images had some visible contrast difference between the blood and myocardium that facilitated the visualization of typically slower blood flow areas such as the cardiac apex and atria. We did not evaluate the methods proposed in these papers extensively using 4D Flow MRI datasets acquired without the use of contrast agents; however, previous studies have focused on segmenting the epicardial, rather than the endocardial wall of the heart chambers, when applied to datasets acquired without contrast [144].

The datasets used in Paper IV were acquired without the use of contrast agents. To account for the lower contrast differences in these images, we made a few changes in the PC-MRCA generation procedure to reduce the dependence of the resulting cadioangiographies on the magnitude component of the 4D Flow MRI datasets. As expected, the blood pool in the resulting images had lower contrast when compared 
to those obtained from datasets acquired with the use of a contrast agent. Nevertheless, the study's results were not visibly affected by this factor, particularly since the segmentation of the average atlas was performed manually.

The use of non-blood pool and blood pool contrasts agents in 4D Flow MRI acquisitions is fairly extensive [31, 145], and positively influences the quality of the resulting images [146, 147]. However, the most common type of contrast agents are gadolinium based, which is contraindicated in patients with renal impairment. Recently, several studies have highlighted the potential of ferumoxytol, an iron replacement product indicated for the treatment of iron deficiency anemia, as a non-toxic contrast agent that could be used in MR imaging. Ferumoxytol has also been tested in 4D Flow MRI acquisitions, where it has achieved promising results [148]. Currently, however, ferumoxytol is not authorized to be used in Europe, and has only been approved for its use as an iron deficiency treatment in the USA. The availability of safer contrast agents intended for MR imaging, and specifically for 4D Flow MRI, would have a positive impact on the feasibility of this acquisition method in the clinical setting.

\subsection{Future Outlook}

Recently, deep neural networks have been successfully used in a great variety of fields, and medical image analysis is not the exception [149]. Several studies based on deep learning have focused on image segmentation and have applied this technique on two- and three-dimensional medical images $[150,151]$. One of the main challenges of deep learning, however, is the requirement of a relatively large set of already labeled data to be used in the process of training the network. The size of the required set can vary greatly and depends on the complexity of the problem and the particular architecture of the network. Specifically in reference to 4D Flow MRI data, manual generation of such a training set would be extremely time consuming Moreover, commonly used straightforward techniques for augmenting the labeled set, such as cropping or rotating the original data, are not directly applicable to these more complex MR images. A possible solution to this problem could be to use the segmentations resulting from applying the technique described in Paper $\mathbf{V}$ to generate a sufficiently large training set.

Using the presented segmentation technique, clinically important features in the 4D Flow MRI data can be readily analyzed and visualized in the heart and large vessels. Automatic geometry guided seeding of pathlines or streamlines can be used to visualize the velocity fields. Hemodynamic parameters such as kinetic energy, peak flow velocity, helicity, pressure, and flow connectivity, among others, can be automatically calculated and visualized for each region of interest. Abnormal flow (e.g. shunt flow between heart chambers, abnormal flow directions through valves, elevated turbulence intensity) could be emphasized in the results. Moreover, the segmentations could also be used for the generation of input data for model-based analysis tools, such as mathematical models of the cardiovascular system [152], a step towards creating fully automated subject-specific models to characterize global 
hemodynamics.

The addition of a tool to automatically generate and visualize all this information would expedite and significantly improve the utility of 4D Flow MRI in the clinical setting. An example of the visualization capabilities of such a tool can be seen in Figure 25, where kinetic energy, turbulent kinetic energy [153], and helicity density were depicted using volume rendering for a patient with aortic valve stenosis.

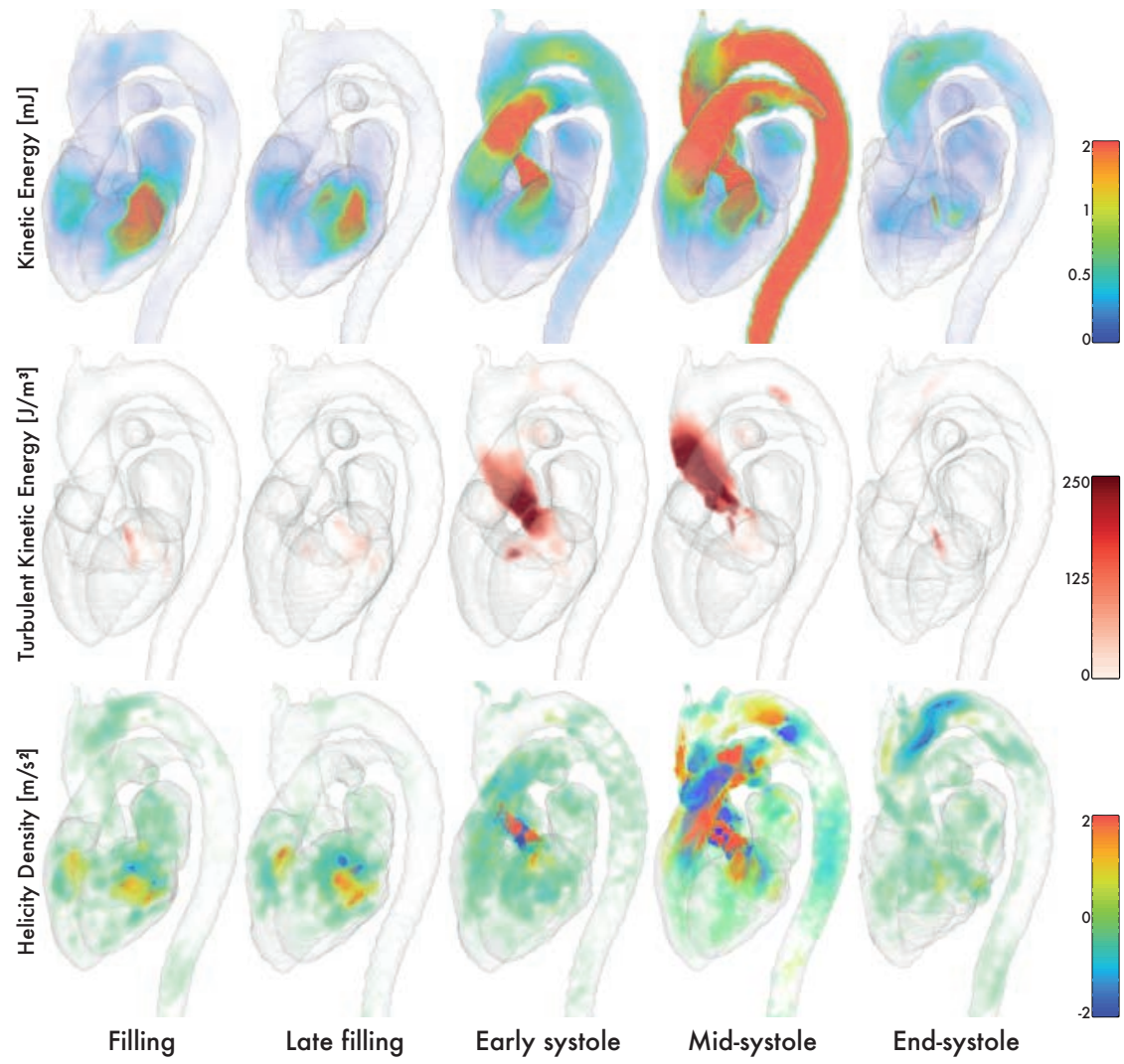

Figure 25: Visualization of hemodynamic parameters using a segmentation generated with the technique presented in Paper V. The dataset belongs to a patient with aortic valve stenosis. 



\section{Bibliography}

[1] Yoram Richter and Elazer R. Edelman. Cardiology is flow. Circulation, 113(23):2679-2682, 2006.

[2] Partho P. Sengupta, Gianni Pedrizzetti, Philip J. Kilner, Arash Kheradvar, Tino Ebbers, Giovanni Tonti, Alan G. Fraser, and Jagat Narula. Emerging trends in CV flow visualization. JACC: Cardiovascular Imaging, 5(3):305316, 2012.

[3] Michael Markl, Philip J Kilner, and Tino Ebbers. Comprehensive 4D velocity mapping of the heart and great vessels by cardiovascular magnetic resonance. Journal of Cardiovascular Magnetic Resonance, 13(1):7, 2011.

[4] R van Pelt, J Olivan Bescos, M Breeuwer, R E Clough, M E Groller, B ter Haar Romeny, and A Vilanova. Interactive Virtual Probing of 4D MRI Blood-Flow. Visualization and Computer Graphics, IEEE Transactions on, 17(12):2153-2162, 2011.

[5] B Köhler, S Born, R F P Van Pelt, U Preim, and B Preim. A Survey of Cardiac 4D PC-MRI Data Processing. Eurographics Workshop on Visual Computing for Biology and Medicine, pages 139-148, 2015.

[6] Walter F Boron and Emile L Boulpaep. Medical physiology: a cellular and molecular approach. W.B. Saunders, 2009.

[7] P J Kilner, G Z Yang, A J Wilkes, R H Mohiaddin, D N Firmin, and $\mathrm{M} H$ Yacoub. Asymmetric redirection of flow through the heart. Nature, 404(6779):759-61, 2000.

[8] Réka Faludi, Mariola Szulik, Jan D’hooge, Paul Herijgers, Frank Rademakers, Gianni Pedrizzetti, and Jens Uwe Voigt. Left ventricular flow patterns in healthy subjects and patients with prosthetic mitral valves: An in vivo study using echocardiographic particle image velocimetry. Journal of Thoracic and Cardiovascular Surgery, 139(6):1501-1510, 2010.

[9] Carl Johan Carlhäll and Ann Bolger. Passing strange: flow in the failing ventricle. Circulation. Heart failure, 3(2):326-331, 2010. 
[10] Jonatan Eriksson, Petter Dyverfeldt, Jan Engvall, Ann F Bolger, Tino Ebbers, and Carl Johan Carlhäll. Quantification of presystolic blood flow organization and energetics in the human left ventricle. American journal of physiology. Heart and circulatory physiology, 300(6):H2135-H2141, 2011.

[11] Jan O. Mangual, Elisabeth Kraigher-Krainer, Alessio De Luca, Loira Toncelli, Amil Shah, Scott Solomon, Giorgio Galanti, Federico Domenichini, and Gianni Pedrizzetti. Comparative numerical study on left ventricular fluid dynamics after dilated cardiomyopathy. Journal of Biomechanics, 46(10):16111617, 2013.

[12] Pim van Ooij, Wouter V Potters, Jeremy Collins, Maria Carr, James Carr, S Chris Malaisrie, Paul W M Fedak, Patrick M McCarthy, Michael Markl, and Alex J Barker. Characterization of abnormal wall shear stress using 4D flow MRI in human bicuspid aortopathy. Annals of biomedical engineering, 43(6):1385-1397, 2015.

[13] Pim van Ooij, Wouter V Potters, Aart J Nederveen, Bradley D Allen, Jeremy Collins, James Carr, S Chris Malaisrie, Michael Markl, and Alex J Barker. A methodology to detect abnormal relative wall shear stress on the full surface of the thoracic aorta using four-dimensional flow MRI. Magnetic Resonance in Medicine, 73(3):1216-1227, 2015.

[14] P G Walker, E M Pedersen, S Oyre, L Flepp, S Ringgaard, R S Heinrich, S P Walton, J M Hasenkam, H S Jorgensen, and A P Yoganathan. Magnetic resonance velocity imaging: a new method for prosthetic heart valve study. The Journal of heart valve disease, 4(3):296-307, 1995.

[15] J Michael Hasenkam, Steffen Ringgaard, Kim Houlind, René M Botnar, Hans Stødkilde-Jørgensen, Peter Boesiger, and Erik Morre Pedersen. Prosthetic heart valve evaluation by magnetic resonance imaging. European Journal of Cardio-Thoracic Surgery, 16(3):300-305, 1999.

[16] Jonatan Eriksson, Carl Carlhäll, Petter Dyverfeldt, Jan Engvall, Ann F Bolger, and Tino Ebbers. Semi-automatic quantification of 4D left ventricular blood flow. Journal of cardiovascular magnetic resonance : official journal of the Society for Cardiovascular Magnetic Resonance, 12(1):9, 2010.

[17] A G Fredriksson, J Zajac, J Eriksson, P Dyverfeldt, A F Bolger, T Ebbers, and C J Carlhäll. 4-D blood flow in the human right ventricle. Am J Physiol Heart Circ Physiol, 301(6):H2344-50, 2011.

[18] Jonatan Eriksson, Ann F Bolger, Tino Ebbers, and Carl-Johan Carlhäll. Fourdimensional blood flow-specific markers of LV dysfunction in dilated cardiomyopathy. European heart journal cardiovascular Imaging, 14(5):417424, 2013. 
[19] Emil Svalbring, Alexandru Fredriksson, Jonatan Eriksson, Petter Dyverfeldt, Tino Ebbers, Ann F Bolger, Jan Engvall, and Carl-Johan Carlhäll. Altered Diastolic Flow Patterns and Kinetic Energy in Subtle Left Ventricular Remodeling and Dysfunction Detected by 4D Flow MRI. PLOS ONE, 11(8):1-12, 2016.

[20] Stephen Gaeta, Petter Dyverfeldt, Jonatan Eriksson, Carl-Johan Carlhäll, Tino Ebbers, and Ann F Bolger. Fixed volume particle trace emission for the analysis of left atrial blood flow using 4D Flow MRI. Magnetic resonance imaging, 47:83-88, 2017.

[21] E Mark Haacke, Robert W Brown, Michael R Thompson, and Ramesh Venkatesan. Magnetic Resonance Imaging: Physical Principles and Sequence Design, volume 1st. Wiley, 1999.

[22] Donald W. McRobbie, Elizabeth A. Moore, Martin J. Graves, and Martin R. Prince. MRI from picture to proton. Cambridge University Press, 2006.

[23] V.S. Lee. Cardiovascular MRI: Physical Principles to Practical Protocols. LWW medical book collection. Lippincott Williams \& Wilkins, 2006.

[24] Klaus Scheffler and Stefan Lehnhardt. Principles and applications of balanced SSFP techniques. European Radiology, 13(11):2409-2418, 2003.

[25] Andrew D Scott, Jennifer Keegan, and David N Firmin. Motion in cardiovascular MR imaging. Radiology, 250(2):331-351, 2009.

[26] Gianni Pedrizzetti and Federico Domenichini. Left Ventricular Fluid Mechanics: The Long Way from Theoretical Models to Clinical Applications. Annals of Biomedical Engineering, 43(1):26-40, 2014.

[27] Paul R. Moran. A flow velocity zeugmatographic interlace for NMR imaging in humans. Magnetic Resonance Imaging, 1(4):197-203, 1982.

[28] N J Pelc, R J Herfkens, A Shimakawa, and D R Enzmann. Phase contrast cine magnetic resonance imaging. Magnetic resonance quarterly, 7(4):229-254, 1991.

[29] Peter D. Gatehouse, Jennifer Keegan, Lindsey A. Crowe, Sharmeen Masood, Raad H. Mohiaddin, Karl-Friedrich Kreitner, and David N. Firmin. Applications of phase-contrast flow and velocity imaging in cardiovascular MRI. European Radiology, 15(10):2172-2184, 2005.

[30] Zoran Stankovic, Bradley D Allen, Julio Garcia, Kelly B Jarvis, and Michael Markl. 4D flow imaging with MRI. Cardiovascular diagnosis and therapy, 4(2):173-92, 2014. 
[31] Petter Dyverfeldt, Malenka Bissell, Alex J. Barker, Ann F. Bolger, Carl-Johan Carlhäll, Tino Ebbers, Christopher J. Francios, Alex Frydrychowicz, Julia Geiger, Daniel Giese, Michael D. Hope, Philip J. Kilner, Sebastian Kozerke, Saul Myerson, Stefan Neubauer, Oliver Wieben, and Michael Markl. 4D flow cardiovascular magnetic resonance consensus statement. Journal of Cardiovascular Magnetic Resonance, 17(1):72, 2015.

[32] Michael D. Hope, Tony Sedlic, and Petter Dyverfeldt. Cardiothoracic Magnetic Resonance Flow Imaging. Journal of Thoracic Imaging, 28(4):217-230, 2013.

[33] Stijntje D Roes, Sebastiaan Hammer, Rob J van der Geest, Nina Ajmone Marsan, Jeroen J Bax, Hildo J Lamb, Johan H C Reiber, Albert de Roos, and Jos J M Westenberg. Flow assessment through four heart valves simultaneously using 3-dimensional 3-directional velocity-encoded magnetic resonance imaging with retrospective valve tracking in healthy volunteers and patients with valvular regurgitation. Investigative radiology, 44(10):669-75, 2009.

[34] Tino Ebbers and Gunnar Farnebäck. Improving computation of cardiovascular relative pressure fields from velocity MRI. Journal of Magnetic Resonance Imaging, 30(1):54-61, 2009.

[35] Petter Dyverfeldt, Michael D. Hope, Elaine E. Tseng, and David Saloner. Magnetic resonance measurement of turbulent kinetic energy for the estimation of irreversible pressure loss in aortic stenosis. JACC: Cardiovascular Imaging, 6(1):64-71, 2013.

[36] Daniel Hirtler, Julio Garcia, Alex J. Barker, and Julia Geiger. Assessment of intracardiac flow and vorticity in the right heart of patients after repair of tetralogy of Fallot by flow-sensitive 4D MRI. European Radiology, pages $1-10,2016$.

[37] Pablo Bächler, Israel Valverde, Natalia Pinochet, Sarah Nordmeyer, Titus Kuehne, Gérard Crelier, Cristián Tejos, Pablo Irarrazaval, Philipp Beerbaum, and Sergio Uribe. Caval Blood Flow Distribution in Patients with Fontan Circulation: Quantification by Using Particle Traces from 4D Flow MR Imaging. Radiology, 267(1):1-14, 2013.

[38] Jasjit S. Suri, Kecheng Liu, Laura Reden, and Swamy Laxminarayan. A review on MR vascular image processing algorithms: Acquisition and prefiltering: Part I. IEEE Transactions on Information Technology in Biomedicine, 6(4):324-337, 2002.

[39] Martin R. Prince, E. Kent Yucel, John A. Kaufman, David C. Harrison, and Stuart C. Geller. Dynamic gadolinium-enhanced three-dimensional abdominal MR arteriography. Journal of Magnetic Resonance Imaging, 3(6):877881, 1993. 
[40] Tomonori Kanda, Toshio Fukusato, Megumi Matsuda, Keiko Toyoda, Hiroshi Oba, Jun'ichi Kotoku, Takahiro Haruyama, Kazuhiro Kitajima, and Shigeru Furui. Gadolinium-based Contrast Agent Accumulates in the Brain Even in Subjects without Severe Renal Dysfunction: Evaluation of Autopsy Brain Specimens with Inductively Coupled Plasma Mass Spectroscopy. Radiology, 276(1):228-232, 2015.

[41] C. L. Dumoulin, S. P. Souza, M. F. Walker, and W. Wagle. Three-dimensional phase contrast angiography. Magnetic Resonance in Medicine, 9(1):139-149, 1989.

[42] C L Dumoulin. Phase contrast MR angiography techniques. Magn Reson Imaging Clin N Am, 3(3):399-411, 1995.

[43] J W M Van Goethem, L van den Hauwe, O Ozsarlak, and P M Parizel. Phasecontrast magnetic resonance angiography. JBR-BTR: organe de la Société royale belge de radiologie (SRBR), 86(6):340-4, 2003.

[44] M Markl, A Harloff, T A Bley, M Zaitsev, B Jung, E Weigang, M Langer, J Hennig, and A Frydrychowicz. Time-resolved 3D MR velocity mapping at 3T: improved navigator-gated assessment of vascular anatomy and blood flow. J Magn Reson Imaging, 25(4):824-831, 2007.

[45] J Bock, O Wieben, K Johnson, J Hennig, and M Markl. Optimal processing to derive static PC-MRA from time-resolved 3D PC-MRI data. In Proceedings 16th Scientific Meeting, International Society for Magnetic Resonance in Medicine, volume Toronto, page 3053, 2008.

[46] A. Hennemuth, O. Friman, C. Schumann, J. Bock, J. Drexl, M. Huellebrand, M. Markl, and H.-O. Peitgen. Fast interactive exploration of 4D MRI flow data. page 79640E. International Society for Optics and Photonics, 2011.

[47] A.P. Dhawan. Medical Image Analysis. IEEE Press Series on Biomedical Engineering. Wiley, 2011.

[48] Daniel Rueckert and Paul Aljabar. Nonrigid registration of medical images: Theory, methods, and applications. In IEEE Signal Processing Magazine, volume 27, pages 113-119, 2010.

[49] A. Ardeshir Goshtasby. Image Registration: Principles, Tools and Methods. Advances in Computer Vision and Pattern Recognition, pages 1-436, 2012.

[50] Aristeidis Sotiras, Christos Davatzikos, and Nikos Paragios. Deformable medical image registration: A survey. IEEE Transactions on Medical Imaging, 32(7):1153-1190, 2013.

[51] G P Penney, J Weese, J a Little, P Desmedt, D L Hill, and D J Hawkes. A comparison of similarity measures for use in 2-D-3-D medical image registration. IEEE transactions on medical imaging, 17(4):586-595, 1998. 
[52] Jorge Nocedal, Stephen J Wright, and SpringerLink (Online service). Numerical Optimization. Springer New York, 2006.

[53] V R S Mani and S Arivazhagan. Survey of Medical Image Registration. Journal of Biomedical Engineering and Technology, 1(2):8-25, 2013.

[54] Francisco P.M. Oliveira and João Manuel R.S. Tavares. Medical image registration: A review. Computer Methods in Biomechanics and Biomedical Engineering, 17(2):73-93, 2014.

[55] Timo Mäkelä, Patrick Clarysse, Outi Sipilä, Nicoleta Pauna, Quoc Cuong Pham, Toivo Katila, and Isabelle E. Magnin. A review of cardiac image registration methods. IEEE Transactions on Medical Imaging, 21(9):10111021, 2002.

[56] Jasbir Sra. Cardiac Image Registration. Journal of Atrial Fibrillation, 1(3):25, 2008.

[57] Xavier Bâty, Christine Cavaro-Ménard, Vincent Roullier, and Jean Jacques Le Jeune. Model based and iconic multimodal registration to merge gated cardiac PET, CT and MR sequences. In Annual International Conference of the IEEE Engineering in Medicine and Biology - Proceedings, pages 852-855, 2007.

[58] Julián Betancur, Antoine Simon, Frédéric Schnell, Erwan Donal, Alfredo Hernández, and Mireille Garreau. Evaluation of a motion artifacts removal approach on breath-hold cine-magnetic resonance images of hypertrophic cardiomyopathy subjects. In IX International Seminar on Medical Information Processing and Analysis, volume 8922, 2013.

[59] Maria Chiara Carminati, Francesco Maffessanti, and Enrico Gianluca Caiani. Nearly automated motion artifacts correction between multi breath-hold shortaxis and long-axis cine CMR images. Computers in Biology and Medicine, 46(1):42-50, 2014.

[60] Raghavendra Chandrashekara, Raad H. Mohiaddin, and Daniel Rueckert. Analysis of 3-D myocardial motion in tagged MR images using nonrigid image registration. IEEE Transactions on Medical Imaging, 23(10):12451250, 2004.

[61] Nikhil S. Phatak, Steve A. Maas, Alexander I. Veress, Nathan A. Pack, Edward V R Di Bella, and Jeffrey A. Weiss. Strain measurement in the left ventricle during systole with deformable image registration. Medical Image Analysis, 13(2):354-361, 2009.

[62] M Lorenzo-Valdés, G Sanchez-Ortiz, R Mohiaddin, and Daniel Rueckert. Atlas-Based Segmentation and Tracking of 3D Cardiac MR Images Using Non-rigid Registration. Medical Image Computing and Computer Assisted Intervention, 2488(Lv):642-650, 2002. 
[63] Wenjia Bai, Wenzhe Shi, Christian Ledig, and Daniel Rueckert. Multi-atlas segmentation with augmented features for cardiac MR images. Medical Image Analysis, 19(1):98-109, 2014.

[64] Pau Medrano-Gracia, Brett R Cowan, Bharath Ambale-Venkatesh, David A Bluemke, John Eng, John Paul Finn, Carissa G Fonseca, Joao AC Lima, Avan Suinesiaputra, and Alistair A Young. Left ventricular shape variation in asymptomatic populations: the multi-ethnic study of atherosclerosis. Journal of Cardiovascular Magnetic Resonance, 16(1):56, 2014.

[65] Jan L. Bruse, Kristin McLeod, Giovanni Biglino, Hopewell N. Ntsinjana, Claudio Capelli, Tain-Yen Hsia, Maxime Sermesant, Xavier Pennec, Andrew M. Taylor, and Silvia Schievano. A statistical shape modelling framework to extract 3D shape biomarkers from medical imaging data: assessing arch morphology of repaired coarctation of the aorta. BMC Medical Imaging, 16(1):40, 2016.

[66] Mark Holden, Derek L.G. Hill, Erika R.E. Denton, Jo M. Jarosz, Tim C.S. Cox, Torsten Rohlfing, Joanne Goodey, and David J. Hawkes. Voxel similarity measures for 3-D serial MR brain image registration. IEEE Transactions on Medical Imaging, 19(2):94-102, 2000.

[67] Ehsan Golkar, Ashrani A.Abd Rahni, and Riza Sulaiman. Comparison of image registration similarity measures for an abdominal organ segmentation framework. In IECBES 2014, Conference Proceedings - IEEE Conference on Biomedical Engineering and Sciences, pages 442-445, 2015.

[68] Frederik Maes, André Collignon, Dirk Vandermeulen, Guy Marchal, and Paul Suetens. Multimodality image registration by maximization of mutual information. IEEE Transactions on Medical Imaging, 16(2):187-198, 1997.

[69] P Viola and W M Wells III. Alignment by Maximization of Mutual Information. The International Journal of Computer Vision, 24(2):137-154, 1997.

[70] P Thévenaz, M Unser, and P. Thevenaz. Optimization of mutual information for multiresolution image registration. IEEE Transactions on Image Processing, 9(12):2083-2099, 2000.

[71] David J. Fleet and Allan D. Jepson. Computation of component image velocity from local phase information. International Journal of Computer Vision, 5(1):77-104, 1990.

[72] Hans Knutsson and Mats Andersson. Morphons: Segmentation using elastic canvas and paint on priors. In Proceedings - International Conference on Image Processing, ICIP, volume 2, pages 1226-1229, 2005. 
[73] Hans Knutsson. Representing local structure using tensors. Conference: Proceedings of 6th Scandinavian Conference on Image Analysis, pages 545556, 1989.

[74] Daniel Forsberg. Robust Image Registration for Improved Clinical Efficiency: Using Local Structure Analysis and Model-Based Processing. PhD thesis, Linköping University Institute of Technology, Center for Medical Image Science and Visualization (CMIV), 2013.

[75] Adriane Parraga, Johanna Pettersson, Altamiro Susin, Mathieu De Craene, and Benoit Macq. Non-rigid registration methods assessment of 3D CT images for head-neck radiotherapy. Proceedings of SPIE, 6512:6-9, 2007.

[76] Olof Dahlqvist Leinhard, Andreas Johansson, Joakim Rydell, Johan Kihlberg, Örjan Smedby, Fredrik H. Nyström, Peter Lundberg, and Magnus Borga. Quantification of abdominal fat accumulation during hyperalimentation using MRI. In ISMRM Annual Meeting, page 206, 2009.

[77] Akihiro Kikuchi, Masahisa Onoguchi, Hiroyuki Horikoshi, Karl Sjostrand, and Lars Edenbrandt. Automated segmentation of the skeleton in whole-body bone scans: influence of difference in atlas. Nuclear medicine communications, 33(9):947-953, 2012.

[78] Nhat Trung Doan, Jonathan Orban de Xivry, and Benoît Macq. Effect of inter-subject variation on the accuracy of atlas-based segmentation applied to human brain structures. volume 7623, pages 1-10, 2010.

[79] Adriane Parraga, Benoit MacQ, and Mathieu De Craene. Anatomical atlas in the context of head and neck radiotherapy and its use to automatic segmentation. Biomedical Signal Processing and Control, 7(5):447-455, 2012.

[80] Ruzena Bajcsy and Stane Kovačič. Multiresolution elastic matching. Computer Vision, Graphics, and Image Processing, 46(1):1-21, 1989.

[81] Gary E. Christensen, Richard D. Rabbitt, and Michael I. Miller. Deformable templates using large deformation kinematics. IEEE Transactions on Image Processing, 5(10):1435-1447, 1996.

[82] Rc Gonzalez and Re Woods. Digital image processing. Pearson Education, 2002.

[83] Milan Sonka, Vaclav Hlavac, and Roger Boyle. Image Processing, Analysis, and Machine Vision. Cengage Learning, 2008.

[84] Rajeshwar Dass and Swapna Devi. Image Segmentation Techniques. International Journal of Electronics \& Communication Technology (IJECT), 3(1):66-70, 2012. 
[85] Nida M. Zaitoun and Musbah J. Aqel. Survey on Image Segmentation Techniques. In Procedia Computer Science, volume 65, pages 797-806, 2015.

[86] Neeraj Sharma and Lalit M Aggarwal. Automated medical image segmentation techniques. Journal of medical physics / Association of Medical Physicists of India, 35(1):3-14, 2010.

[87] Saleha Masood, Muhammad Sharif, Afifa Masood, Mussarat Yasmin, and Mudassar Raza. A Survey on Medical Image Segmentation. Current Medical Imaging Reviews, 11(1):3-14, 2015.

[88] F. Kirbas, C. Quek. A review of vessel extraction techniques and algorithms. Computing Surveys, 36(2):81-121, 2004.

[89] David Lesage, Elsa D. Angelini, Isabelle Bloch, and Gareth Funka-Lea. A review of 3D vessel lumen segmentation techniques: Models, features and extraction schemes. Medical Image Analysis, 13(6):819-845, 2009.

[90] Caroline Petitjean and Jean Nicolas Dacher. A review of segmentation methods in short axis cardiac MR images. Medical Image Analysis, 15(2):169-184, 2011.

[91] Torsten Rohlfing, Robert Brandt, Randolf Menzel, Daniel B. Russakoff, and Calvin R. Maurer Jr. Quo Vadis, Atlas-Based Segmentation? In Handbook of Biomedical Image Analysis, chapter 11, pages 435-486. Springer US, 2005.

[92] Juan Eugenio Iglesias and Mert R. Sabuncu. Multi-atlas segmentation of biomedical images: A survey. Medical Image Analysis, 24(1):205-219, 2015.

[93] Torsten Rohlfing, Robert Brandt, Randolf Menzel, and Calvin R. Maurer. Evaluation of atlas selection strategies for atlas-based image segmentation with application to confocal microscopy images of bee brains. NeuroImage, 21(4):1428-1442, 2004.

[94] Jasjit S Suri and David L Wilson. Handbook of Biomedical Image Analysis Volume III: Registration Models. Springer, 2005.

[95] P. Aljabar, R. A. Heckemann, A. Hammers, J. V. Hajnal, and D. Rueckert. Multi-atlas based segmentation of brain images: Atlas selection and its effect on accuracy. NeuroImage, 46(3):726-738, 2009.

[96] Mariano Cabezas, Arnau Oliver, Xavier Lladó, Jordi Freixenet, and Meritxell Bach Cuadra. A review of atlas-based segmentation for magnetic resonance brain images. Computer Methods and Programs in Biomedicine, 104(3), 2011.

[97] Thomas Robin Langerak, Uulke A. Van Der Heide, Alexis N T J Kotte, Max A. Viergever, Marco Van Vulpen, and Josien P W Pluim. Label fusion in atlas-based segmentation using a selective and iterative method for 
performance level estimation (SIMPLE). IEEE Transactions on Medical Imaging, 29(12):2000-2008, 2010.

[98] X. Zhuang, K. Leung, K. Rhode, R. Razavi, D. Hawkes, and S. Ourselin. Whole heart segmentation of cardiac MRI using multiple path propagation strategy. In Lecture Notes in Computer Science, volume 6361 LNCS, pages 435-443, 2010.

[99] M. A. Zuluaga, A. Mendelson, M. J. Cardoso, A. M. Taylor, and S. Ourselin. Multi-atlas based pathological stratification of D-TGA congenital heart disease. In 2014 IEEE 11th International Symposium on Biomedical Imaging (ISBI), pages 109-112, 2014.

[100] Anette Karlsson, Johannes Rosander, Thobias Romu, Joakim Tallberg, Anders Grönqvist, Magnus Borga, and Olof Dahlqvist Leinhard. Automatic and quantitative assessment of regional muscle volume by multi-atlas segmentation using whole-body water-fat MRI. Journal of Magnetic Resonance Imaging, 41(6):1558-1569, 2015.

[101] Albert Gubern-Mérida, Michiel Kallenberg, Robert Martí, and Nico Karssemeijer. Segmentation of the Pectoral Muscle in Breast MRI Using AtlasBased Approaches. In International Conference on Medical Image Computing and Computer-Assisted Intervention (MICCAI), volume 15, pages 371378. Springer Berlin Heidelberg, 2012.

[102] Liang Shan, Christopher Zach, Cecil Charles, and Marc Niethammer. Automatic atlas-based three-label cartilage segmentation from MR knee images. Medical Image Analysis, 18(7):1233-1246, 2014.

[103] Simon K. Warfield, Kelly H. Zou, and William M. Wells. Simultaneous truth and performance level estimation (STAPLE): An algorithm for the validation of image segmentation. IEEE Transactions on Medical Imaging, 23(7):903$921,2004$.

[104] Ivana Išgum, Marius Staring, Annemarieke Rutten, Mathias Prokop, Max A. Viergever, and Bram Van Ginneken. Multi-atlas-based segmentation with local decision fusion-application to cardiac and aortic segmentation in CT scans. IEEE Transactions on Medical Imaging, 28(7):1000-1010, 2009.

[105] Mert R Sabuncu, B T Thomas Yeo, K Van Leemput, Bruce Fischl, and Polina Golland. A Generative Model for Image Segmentation Based on Label Fusion. IEEE Transactions on Medical Imaging, 29(10):1714-1729, 2010.

[106] Jyrki M P Lötjönen, Robin Wolz, Juha R Koikkalainen, Lennart Thurfjell, Gunhild Waldemar, Hilkka Soininen, and Daniel Rueckert. Fast and robust multi-atlas segmentation of brain magnetic resonance images. NeuroImage, 49(3):2352-2365, 2010. 
[107] Andrew J. Asman and Bennett A. Landman. Non-local statistical label fusion for multi-atlas segmentation. Medical Image Analysis, 17(2):194-208, 2013.

[108] Wenjia Bai, Wenzhe Shi, Declan P. O’Regan, Tong Tong, Haiyan Wang, Shahnaz Jamil-Copley, Nicholas S. Peters, and Daniel Rueckert. A probabilistic patch-based label fusion model for multi-atlas segmentation with registration refinement: Application to cardiac MR images. IEEE Transactions on Medical Imaging, 32(7):1302-1315, 2013.

[109] Hongzhi Wang, Jung W. Suh, Sandhitsu R. Das, John B. Pluta, Caryne Craige, and Paul A. Yushkevich. Multi-atlas segmentation with joint label fusion. IEEE Transactions on Pattern Analysis and Machine Intelligence, 35(3):611623, 2013.

[110] Avan Suinesiaputra, Brett R Cowan, Ahmed O Al-Agamy, Mustafa A Elattar, Nicholas Ayache, Ahmed S Fahmy, Ayman M Khalifa, Pau Medrano-Gracia, Marie-Pierre Jolly, Alan H Kadish, Daniel C Lee, Ján Margeta, Simon K Warfield, and Alistair A Young. A collaborative resource to build consensus for automated left ventricular segmentation of cardiac MR images. Medical Image Analysis, 18(1):50-62, 2014.

[111] Avan Suinesiaputra, David A. Bluemke, Brett R. Cowan, Matthias G. Friedrich, Christopher M. Kramer, Raymond Kwong, Sven Plein, et al. Quantification of LV function and mass by cardiovascular magnetic resonance: multi-center variability and consensus contours. Journal of Cardiovascular Magnetic Resonance, 17(1):63, 2015.

[112] A. P. Dempster, N. M. Laird, and D. B. Rubin. Maximum Likelihood from Incomplete Data via the EM Algorithm. Journal of the Royal Statistical Society. Series B, 39(1):1-38, 1977.

[113] Abdel Aziz Taha and Allan Hanbury. Metrics for evaluating 3D medical image segmentation: Analysis, selection, and tool. BMC Medical Imaging, 15(1), 2015.

[114] Lee R. Dice. Measures of the Amount of Ecologic Association Between Species. Ecology, 26(3):297-302, 1945.

[115] F Hausdorff. Set theory. American Mathematical Society, pages 166-167, 1957.

[116] Roy Van Pelt, Javier Oliván Bescós, Marcel Breeuwer, Rachel E. Clough, M. Eduard Gröller, Bart Ter Haar Romenij, and Anna Vilanova. Exploration of 4D MRI blood flow using stylistic visualization. IEEE Transactions on Visualization and Computer Graphics, 16(6):1339-1347, 2010. 
[117] Robert V. Bergen, Hung Yu Lin, Murray E. Alexander, and Christopher P. Bidinosti. 4D MR phase and magnitude segmentations with GPU parallel computing. Magnetic Resonance Imaging, 33(1):134-145, 2015.

[118] Paola Volonghi, Daniele Tresoldi, Marcello Cadioli, Antonio M. Usuelli, Raffaele Ponzini, Umberto Morbiducci, Antonio Esposito, and Giovanna Rizzo. Automatic extraction of three-dimensional thoracic aorta geometric model from phase contrast MRI for morphometric and hemodynamic characterization. Magnetic Resonance in Medicine, 75(2):873-882, 2016.

[119] Benjamin Köhler, Uta Preim, Matthias Grothoff, Matthias Gutberlet, Katharina Fischbach, and Bernhard Preim. Robust Cardiac Function Assessment in 4D PC-MRI Data of the Aorta and Pulmonary Artery. In Computer Graphics Forum, volume 35, pages 32-43, 2016.

[120] Benjamin Köhler, Uta Preim, Matthias Grothoff, Matthias Gutberlet, Katharina Fischbach, and Bernhard Preim. Motion-aware stroke volume quantification in 4D PC-MRI data of the human aorta. International Journal of Computer Assisted Radiology and Surgery, 11(2):169-179, 2016.

[121] Kate McLeish, Derek L.G. Hill, David Atkinson, Jane M. Blackall, and Reza Razavi. A study of the motion and deformation of the heart due to respiration. IEEE Transactions on Medical Imaging, 21(9):1142-1150, 2002.

[122] Nikos Paragios. A variational approach for the segmentation of the left ventricle in cardiac image analysis. International Journal of Computer Vision, 50(3):345-362, 2002.

[123] Honghai Zhang, Andreas Wahle, Ryan K. Johnson, Thomas D. Scholz, and Milan Sonka. 4-D cardiac MR image analysis: Left and right ventricular morphology and function. IEEE Transactions on Medical Imaging, 29(2):350$364,2010$.

[124] Maria Lorenzo-Valdés, Gerardo I. Sanchez-Ortiz, Andrew G. Elkington, Raad H. Mohiaddin, and Daniel Rueckert. Segmentation of 4D cardiac MR images using a probabilistic atlas and the EM algorithm. Medical Image Analysis, 8(3):255-265, 2004.

[125] Peng Peng, Karim Lekadir, Ali Gooya, Ling Shao, Steffen E. Petersen, and Alejandro F. Frangi. A review of heart chamber segmentation for structural and functional analysis using cardiac magnetic resonance imaging. Magnetic Resonance Materials in Physics, Biology and Medicine, 29(2):155-195, 2016.

[126] Michael H. Buonocore. Visualizing blood flow patterns using streamlines, arrows, and particle paths. Magnetic Resonance in Medicine, 40(2):210-226, 1998. 
[127] Lars Wigström, Tino Ebbers, Anna Fyrenius, Matts Karlsson, Jan Engvall, Bengt Wranne, and Ann F. Bolger. Particle trace visualization of intracardiac flow using time-resolved 3D phase contrast MRI. Magnetic Resonance in Medicine, 41(4):793-799, 1999.

[128] Daniel Rodriguez Muñoz, Michael Markl, José Luis Moya Mur, Alex Barker, Covadonga Fernández-Golfín, Patrizio Lancellotti, and José Luis Zamorano Gómez. Intracardiac flow visualization: Current status and future directions. European Heart Journal Cardiovascular Imaging, 14(11):1029-1038, 2013.

[129] Jakub Zajac, Jonatan Eriksson, Petter Dyverfeldt, Ann F. Bolger, Tino Ebbers, and Carl Johan Carlhäll. Turbulent kinetic energy in normal and myopathic left ventricles. Journal of Magnetic Resonance Imaging, 41(4):1021-1029, 2015.

[130] Mohammed S M Elbaz, Emmeline E Calkoen, Jos J M Westenberg, Boudewijn P F Lelieveldt, Arno A W Roest, and Rob J van der Geest. Vortex flow during early and late left ventricular filling in normal subjects: quantitative characterization using retrospectively-gated 4D flow cardiovascular magnetic resonance and three-dimensional vortex core analysis. Journal of Cardiovascular Magnetic Resonance, 16(1):78, 2014.

[131] Michael D. Hope, Jarrett Wrenn, Monica Sigovan, Elyse Foster, Elaine E. Tseng, and David Saloner. Imaging biomarkers of aortic disease: Increased growth rates with eccentric systolic flow. Journal of the American College of Cardiology, 60(4):356-357, 2012.

[132] Alexandru Grigorescu Fredriksson, Emil Svalbring, Jonatan Eriksson, Petter Dyverfeldt, Urban Alehagen, Jan Engvall, Tino Ebbers, and Carl Johan Carlhäll. 4D flow MRI can detect subtle right ventricular dysfunction in primary left ventricular disease. Journal of Magnetic Resonance Imaging, 43(3):558-565, 2016.

[133] Yoshie Nogami, Tomoko Ishizu, Akiko Atsumi, Masayoshi Yamamoto, Ryo Kawamura, Yoshihiro Seo, and Kazutaka Aonuma. Abnormal early diastolic intraventricular flow 'kinetic energy index' assessed by vector flow mapping in patients with elevated filling pressure. European Heart Journal Cardiovascular Imaging, 14(3):253-260, 2013.

[134] Nadya Al-Wakeel, Joao Filipe Fernandes, Aref Amiri, Henryk Siniawski, Leonid Goubergrits, Felix Berger, and Titus Kuehne. Hemodynamic and energetic aspects of the left ventricle in patients with mitral regurgitation before and after mitral valve surgery. Journal of Magnetic Resonance Imaging, 42(6):1705-1712, 2015.

[135] R. Lorenz, J. Bock, A. J. Barker, F. Von Knobelsdorff-Brenkenhoff, W. Wallis, J. G. Korvink, M. M. Bissell, J. Schulz-Menger, and M. Markl. 4D flow 
magnetic resonance imaging in bicuspid aortic valve disease demonstrates altered distribution of aortic blood flow helicity. Magnetic Resonance in Medicine, 71(4):1542-1553, 2014.

[136] Julio Garcia, Alex J. Barker, Jeremy D. Collins, James C. Carr, and Michael Markl. Volumetric quantification of absolute local normalized helicity in patients with bicuspid aortic valve and aortic dilatation. Magnetic Resonance in Medicine, 78(2):689-701, 2017.

[137] J P Lewis. Fast Normalized Cross-Correlation. Vision Interface, 1995(1):1-7, 1995.

[138] Stefan Klein, Marius Staring, Keelin Murphy, Max A. Viergever, and Josien P W Pluim. Elastix: A toolbox for intensity-based medical image registration. IEEE Transactions on Medical Imaging, 29(1):196-205, 2010.

[139] Albert Hsiao, Marcus T. Alley, Payam Massaband, Robert J. Herfkens, Frandics P. Chan, and Shreyas S. Vasanawala. Improved cardiovascular flow quantification with time-resolved volumetric phase-contrast MRI. Pediatric Radiology, 41(6):711-720, 2011.

[140] Israel Valverde, Sarah Nordmeyer, Sergio Uribe, Gerald Greil, Felix Berger, Titus Kuehne, and Philipp Beerbaum. Systemic-to-pulmonary collateral flow in patients with palliated univentricular heart physiology: Measurement using cardiovascular magnetic resonance 4D velocity acquisition. Journal of Cardiovascular Magnetic Resonance, 14(1), 2012.

[141] Umar Tariq, Albert Hsiao, Marcus Alley, Tao Zhang, Michael Lustig, and Shreyas S. Vasanawala. Venous and arterial flow quantification are equally accurate and precise with parallel imaging compressed sensing 4D phase contrast MRI. Journal of Magnetic Resonance Imaging, 37(6):1419-1426, 2013.

[142] Tobias Heimann and Hans Peter Meinzer. Statistical shape models for 3D medical image segmentation: A review. Medical Image Analysis, 13(4):543$563,2009$.

[143] Sandro Queiros, Daniel Barbosa, Brecht Heyde, Pedro Morais, Joao L Vilaca, Denis Friboulet, Olivier Bernard, and Jan D'hooge. Fast automatic myocardial segmentation in 4D cine CMR datasets. Medical image analysis, 18(7):1115-1131, 2014.

[144] Rahil Shahzad, Daniel Bos, Ricardo P.J. Budde, Karlijn Pellikaan, Wiro J. Niessen, Aad Van Der Lugt, and Theo Van Walsum. Automatic segmentation and quantification of the cardiac structures from non-contrast-enhanced cardiac CT scans. Physics in Medicine and Biology, 62(9):3798-3813, 2017. 
[145] Shreyas S Vasanawala, Kate Hanneman, Marcus T Alley, and Albert Hsiao. Congenital heart disease assessment with 4D flow MRI. Journal of magnetic resonance imaging : JMRI, 42(4):870-86, 2015.

[146] Jelena Bock, Alex Frydrychowicz, Aurélien F. Stalder, Thorsten A. Bley, Hans Burkhardt, Jürgen Hennig, and Michael Markl. 4D phase contrast MRI at $3 \mathrm{~T}$ : Effect of standard and blood-pool contrast agents on SNR, PC-MRA, and blood flow visualization. Magnetic Resonance in Medicine, 63(2):330$338,2010$.

[147] Aaron T Hess, Malenka M Bissell, Ntobeko A B Ntusi, Andrew J M Lewis, Elizabeth M Tunnicliffe, Andreas Greiser, Aurelien F Stalder, Jane M Francis, Saul G Myerson, Stefan Neubauer, and Matthew D Robson. Aortic 4D flow: quantification of signal-to-noise ratio as a function of field strength and contrast enhancement for 1.5T, 3T, and 7T. Magnetic resonance in medicine, 73(5):1864-1871, 2015.

[148] Joseph Y. Cheng, Kate Hanneman, Tao Zhang, Marcus T. Alley, Peng Lai, Jonathan I. Tamir, Martin Uecker, John M. Pauly, Michael Lustig, and Shreyas S. Vasanawala. Comprehensive motion-compensated highly accelerated 4D flow MRI with ferumoxytol enhancement for pediatric congenital heart disease. Journal of Magnetic Resonance Imaging, 43(6):1355-1368, 2016.

[149] Geert Litjens, Thijs Kooi, Babak Ehteshami Bejnordi, Arnaud Arindra Adiyoso Setio, Francesco Ciompi, Mohsen Ghafoorian, Jeroen A.W.M. van der Laak, Bram van Ginneken, and Clara I. Sánchez. A survey on deep learning in medical image analysis, 2017.

[150] Rudra P.K. Poudel, Pablo Lamata, and Giovanni Montana. Recurrent fully convolutional neural networks for multi-slice MRI cardiac segmentation. In Lecture Notes in Computer Science, volume 10129 LNCS, pages 83-94, 2017.

[151] Konstantinos Kamnitsas, Christian Ledig, Virginia F.J. Newcombe, Joanna P. Simpson, Andrew D. Kane, David K. Menon, Daniel Rueckert, and Ben Glocker. Efficient multi-scale 3D CNN with fully connected CRF for accurate brain lesion segmentation. Medical Image Analysis, 36:61-78, 2017.

[152] Belén Casas, Jonas Lantz, Federica Viola, Gunnar Cedersund, Ann F. Bolger, Carl Johan Carlhäll, Matts Karlsson, and Tino Ebbers. Bridging the gap between measurements and modelling: A cardiovascular functional avatar. Scientific Reports, 7(1), 2017.

[153] Petter Dyverfeldt, J. P. E. Kvitting, Andreas Sigfridsson, Jan Engvall, Ann F. Bolger, and Tino Ebbers. Assessment of fluctuating velocities in disturbed cardiovascular blood flow: In vivo feasibility of generalized phase-contrast MRI. Journal of Magnetic Resonance Imaging, 28(3):655-663, 2008. 


\section{Papers}

The papers associated with this thesis have been removed for copyright reasons. For more details about these see:

http://urn.kb.se/resolve?urn=urn:nbn:se:liu:diva-145729 